\title{
ANALYSIS ON ANALYTIC SPACES AND NON-SELF-DUAL YANG-MILLS FIELDS
}

\author{
BY
}

\author{
N. P. BUCHDAHL
}

\begin{abstract}
This paper gives a cohomological description of the Witten-IsenbergYasskin-Green generalization to the non-self-dual case of Ward's twistor construction for self-dual Yang-Mills fields. The groundwork for this description is presented in Part I: with a brief introduction to analytic spaces and differential forms thereon, it contains an investigation of the exactness of the holomorphic relative de Rham complex on formal neighbourhoods of submanifolds, results giving sufficient conditions for the invertibility of pull-back functors on categories of analytic objects, and a discussion of the extension problem for analytic objects in the context of the formalism earlier introduced. Part II deals with non-self-dual Yang-Mills fields: the Yang-Mills field and current are identified in terms of the Griffiths obstructions to extension, including a proof of Manin's result that "current = obstruction to third order". All higher order obstructions are identified, there being at most $N^{2}$ for a bundle of rank $N$. An ansatz for producing explicit examples of non-self-dual fields is obtained by using the correspondence. This ansatz generates $\operatorname{SL}(2, \mathbb{C})$ solutions with topological charge 1 on $S^{4}$.
\end{abstract}

Introduction. As the title suggests, the paper is divided into two parts, the first of which lays the mathematical groundwork for the second. Part II, "Non-self-dual Yang-Mills fields", is concerned with the generalization of Ward's twistor construction for self-dual Yang-Mills fields (Ward [28]) to the non-self-dual case, due independently to Witten [30] and Isenberg, Yasskin and Green [19], and with the subsequent cohomological formulation of this generalization by Manin [22].

In broad outline Ward's construction gives a one-to-one correspondence between holomorphic vector bundles with self-dual connection on a complex 4-manifold $X$ and holomorphic vector bundles on an associated complex 3-manifold $Y$ satisfying a certain triviality condition. The term "self-dual" means that the curvature $\mathbf{F}$ of the connection satisfies $* \mathbf{F}=\mathbf{F}$, where $*$ is the Hodge $*$-operator of a metric on $X$; any such connection automatically satisfies the Yang-Mills equations $\nabla * \mathbf{F}=0$. With the imposition of certain conditions on the bundle on $Y$, the bundle and connection on $X$ can be reduced to a $G$-bundle and connection on restriction to real submanifolds for subgroups $G$ of $\operatorname{GL}(N, \mathbb{C})$ : the Ward correspondence subsequently led to the construction by Atiyah, Drinfeld, Hitchin and Manin [2] of all self-dual $\operatorname{SU}(n)$ solutions of the Yang-Mills equations on $S^{4}$, the so-called instantons. It is an unsolved problem as to whether or not there exist strictly non-self-dual SU(2) or

Received by the editors May 25, 1984.

1980 Mathematics Subject Classification. Primary 32C99, 81E10; Secondary 32D15, 58A12.

C(C1985 American Mathematical Society $0002-9947 / 85 \$ 1.00+\$ .25$ per page 
SU(3) solutions on $S^{4}$. If they do exist, they can be nowhere "near" the instantons (Bourguignon and Lawson [4]).

Ward's construction is an example of a technique central to twistor theory which might be called "the method of double fibrations", a transform procedure for establishing correspondences between sets of analytic objects existing, respectively, on $X$ and $Y$ ("object" meaning bundle, cohomology class, mapping, etc.). Underlying this procedure is a holomorphic double fibration

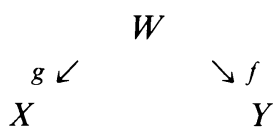

where $f$ and $g$ are suitable holomorphic mappings and $W$ is some intermediary manifold. An object on $Y$ is pulled-back to $W$ by $f$ and subsequently pushed down to $X$ by $g$, the operations of pulling-back and pushing down being achieved by some prescribed mechanism. By virtue of this process the derived object on $X$ should possess some differential property - a section of a bundle satisfying a differential equation, a bundle admitting a connection, a distribution being integrable, etc.reflecting the fact that the intermediary object on $W$ from which it is derived is in some sense constant along the fibres of $f$. Under certain circumstances, the transform is invertible, and the correspondence thus established provides a way of studying the objects on $X$ possessing this differential property in terms of the objects on $Y$ about which one (ideally) has greater knowledge or which are at least free of differential constraints. In this way twistor theory aims of describe physical objects such as fields and particles on space-time in terms of complex analytic objects on a completely different complex manifold.

This transform procedure first appeared in twistor theory in the form of contour integral formulae for massless fields given by Penrose in [24], although the underlying double fibration appears in that paper only implicitly. With the advent of a cohomological interpretation of these formulae the double fibration was made explicit: a complete description of the method transforming elements of certain cohomology groups into solutions of the massless field equations - the so-called Penrose transform - is given in Eastwood, Penrose and Wells [10]. The other standard example of the method applied in twistor theory is Ward's construction already cited, but there are many others, the construction of solutions of the Bogomolny equations (Ward [29]) to name but one. See also Eastwood [9] for further applications.

By replacing $W$ and $Y$ with different manifolds, Witten, Isenberg, Yasskin and Green generalized Ward's correspondence by exhibiting a one-to-one correspondence between a class of holomorphic vector bundles on (the new, now 5-dimensional) $Y$ and holomorphic vector bundles with connection on $X$, but now with no restrictions on the curvature. Using explicit power series calculations, they showed how to determine the field $\mathbf{F}$ from the bundle on $Y$ and proved the important result that the Yang-Mills current $\mathbf{J}=\nabla * \mathbf{F}$ is zero iff the bundle on $Y$ has an extension to the third formal neighbourhood of $Y$ in another associated manifold. (These calculations appear in [18].) 
In [22] Manin cast these results in an invariant form: by using the obstruction theory of Griffiths [14], he identified the field and current in terms of certain cohomology classes associated with the bundle on $Y$, although for his description of the current as the obstruction to extending the bundle to third order, he was forced to rely on the crucial result of Witten, Isenberg, Yasskin and Green which, as he stated in his paper, was clearly a drawback. He subsequently overcame the problem using "Doulbeault cohomology with nilpotents" [23]. Pool [26] used explicit power series calculations to obtain the same results.

An alternative way to overcome such current problems is to generalize the method itself; namely, to allow any or all of the spaces of the double fibration to be analytic spaces rather than just complex manifolds. The method generalizes very naturally to such spaces, and within this framework the complete Yang-Mills construction takes a fairly transparent form. The machinery required for this generalization is developed in Part I: a brief introduction to analytic spaces is given in $\$ 1.1$; and the notion of differential forms on such objects is introduced in $\$ 1.2$. $\$ 1.3$ is concerned with the relative de Rham complex on a particular type of analytic space, the aim of this section being to generalize the classical (relative) de Rham theorem on manifolds to these spaces, subsequently used to express conditions of "constancy along fibres". In $\$ 1.4$ sufficient conditions for inverting holomorphic pull-back functors (and hence the generalized transform) are obtained. Part I concludes in $\$ 1.5$ with a discussion of the extension problem for analytic objects in terms of Griffiths obstructions and the formalism of differential analysis on analytic spaces.

Part $I$ is presented in considerably more generality than is required for the applications of Part II. Apart from inherent mathematical interest, this is motivated by the potential applications of the method to other areas of twistor theory or mathematics, and this generality does not demand a great deal more work than would othewise be required.

Part II commences with a brief account of the facts about complex flag manifolds needed to apply the results of Part I to Yang-Mills theory, these being the particular spaces used in the double fibration for the Witten-Isenberg-Yasskin-Green correspondence. This correspondence is described explicitly in $\$ 2.2$ together with the accompanying transform for cohomology. $\$ \S 2.3$ and 2.4 are devoted, respectively, to the identification of the Yang-Mills field and current in terms of the Griffiths obstructions discussed in 1.5. In $\$ 2.5$ all of the higher order obstructions are identified. It is also shown that if the bundle on $Y$ has rank $N$ and extends to the $\left(N^{2}+2\right)$ th formal neighbourhood, then it extends to all other neighbourhoods also. The paper concludes in $\$ 2.6$ with some examples and an ansatz derived from the correspondence generating explicit (strictly) non-self-dual solutions. This ansatz produces $\operatorname{SL}(2, \mathbb{C})$ solutions on $S^{4}$ with topological charge (i.e. minus the second Chern class) 1 .

I have tried to make the paper relatively complete in order that it might be read by mathematicians and mathematical physicists, particularly those not already familiar with twistor theory. A knowledge of basic complex analysis in several variables, most of which can be found in standard texts such as Hörmander [16], is assumed. Since 
the paper deals exclusively with complex analytic objects, the adjectives "holomorphic" and "analytic" have usually been suppressed: thus, "bundle", for example, means "holomorphic bundle".

Portions of this paper were written over the past two years. A large part was written while I was a student at the Mathematical Institute, Oxford, from 1979-1982; I wish to thank the Royal Commission for the Exhibition of 1851 for finanical support. I also wish to thank my supervisor, Dr. M. G. Eastwood, for his guidance during that period. Most of the remaining research and some of the writing was done at the Institut des Hautes Études Scientifiques from 1982-1983. I am very grateful to IHES for its financial support during that time.

\section{PART I: ANALYSIS ON ANALYTIC SPACES}

1.1. Analytic spaces. The central ideas of this article rest on the ability to "transport" sheaves from one space to another via a mapping of some form, so the two basic mechanisms by which this is achieved will first be recalled before launching into a discussion of analytic spaces proper.

Let $f: X \rightarrow Y$ be a continuous mapping of topological spaces. If $\mathscr{S}$ is a sheaf on $Y$, the topological inverse image $f^{-1} \mathscr{S}$ is the sheaf on $X$ defined by the presheaf

$$
X \supset U \text { open } \mapsto \Gamma(f(U), \mathscr{S})
$$

with the obvious restriction maps. It is characterized by the property that $\left(f^{-1} \mathscr{S}\right)_{x}$ $=\mathscr{S}_{f(x)}$ for all $x \in X$. If $\mathscr{S}$ is a sheaf of groups (resp. rings), so is $f^{-1} \mathscr{S}$; in particular, $f^{-1} \mathscr{C}_{Y}$ is the sheaf of germs of continuous functions on $X$ which are constant along the fibres of $f$. If $\mathscr{S}$ is a sheaf of $\mathscr{C}_{Y}$-modules, $f^{-1} \mathscr{S}$ is a sheaf of $f^{-1} \mathscr{C}_{Y}$-modules, and $f^{-1}$ preserves exact sequences. There is a canonical injection $\Gamma(Y, \mathscr{S}) \rightarrow \Gamma\left(X, f^{-1} \mathscr{S}\right)$ which is an isomorphism if the fibres of $f$ are connected, and if $\mathscr{S}$ is a sheaf of abelian groups, then there are canonical homomorphisms $H^{p}(Y, \mathscr{S}) \rightarrow$ $H^{p}\left(X, f^{-1} \mathscr{S}\right)$.

Going the other way, if $\mathscr{R}$ is a sheaf of abelian groups on $X$, the $q$ th direct image sheaf $f_{*}^{q} \mathscr{R}$ is the sheaf of abelian groups on $Y$ defined by the presheaf

$$
Y \supset U \text { open } \mapsto H^{q}\left(f^{-1}(U), \mathscr{R}\right),
$$

again with the obvious restriction maps. The sheaf $f_{*}^{0} \mathscr{R}$ will always be denoted by $f_{*} \mathscr{R}$, as is customary. $f_{*}^{q}$ is a functor from the category of sheaves of $\mathscr{C}_{X}$-modules to the category of $\mathscr{C}_{Y}$-modules. There is a spectral sequence, called the Leray spectral sequence,

$$
E_{2}^{p, q}=H^{p}\left(Y, f_{*}^{q} \mathscr{R}\right) \Rightarrow H^{p+q}(X, \mathscr{R})
$$

relating the cohomology of $\mathscr{R}$ to that of its direct images (Godement [12]).

Having stated these definitions, we can now give the basic definitions and notions of analytic spaces relevant to this article. Much of the following is from Fischer [11], where further details may be found.

A $\mathbb{C}$-ringed space $X$ is a pair $\left(|X|, \mathcal{O}_{X}\right)$ consisting of a topological space $|X|$ and a sheaf $\mathcal{O}_{X}$ of local $\mathbb{C}$-algebras on $|X|$ such that $\mathcal{O}_{X, x} / \mathbf{m}_{x} \simeq \mathbb{C}$ for each $x \in|X|$, where $\mathbf{m}_{x}$ denotes the unique maximal ideal of the stalk $\mathcal{O}_{X, x}$. A morphism $f: X \rightarrow Y$ of $\mathbb{C}$-ringed spaces is a pair $(|f|, \tilde{f})$ consisting of a continuous map $|f|:|X| \rightarrow|Y|$ and a 
homomorphism of sheaves of $\mathbb{C}$-algebras $\tilde{f}: \mathcal{O}_{Y} \rightarrow|f|_{*} \mathcal{O}_{X}$. By defining composition of morphisms the obvious way, we see that $\mathbb{C}$-ringed spaces form a category.

If $U$ is an open subset of $\mathbb{C}^{n}$ and $I \subset \mathcal{O}_{U}$ is a coherent sheaf of ideals, the space $\left(\operatorname{supp} \mathcal{O}_{U} / I, \mathcal{O}_{U} /\left.I\right|_{\text {supp } \mathcal{O}_{U} / I}\right.$ ) is a $\mathbb{C}$-ringed space ("supp" meaning the point set where the sheaf is nonzero) called a local model. An analytic space $X$ is a $\mathbb{C}$-ringed space such that $|X|$ is Hausdorff and each $x \in|X|$ has a neighbourhood $|U| \subset|X|$ with $\left(|U|,\left.\mathcal{O}_{X \mid}\right|_{|U|}\right)$ isomorphic (as a $\mathbb{C}$-ringed space) to some local model. $\mathbb{C}$-ringed space morphisms between analytic spaces are called holomorphic mappings, and, as might be expected, the set of holomorphic mappings $X \rightarrow \mathbb{C}$ is in 1-1 correspondence with $\Gamma\left(|X|, \mathcal{O}_{X}\right)$.

An analytic sheaf on an analytic space $X$ is a sheaf of $\mathcal{O}_{X}$-modules on $|X|$. An analytic sheaf $\mathscr{S}$ is coherent (resp. locally free) if each $x \in|X|$ has a neighbourhood $|U| \subset|X|$ for which there is an exact sequence

$$
\left.\left.\mathcal{O}_{X}^{p}\right|_{|U|} \rightarrow \mathcal{O}_{X}^{q}\right|_{|U|} \rightarrow \mathscr{S}_{|U|} \rightarrow 0 \quad\left(\text { resp. }\left.\left.\mathcal{O}_{X}^{q}\right|_{|U|} \simeq \mathscr{S}\right|_{|U|}\right) \text {. }
$$

An analytic space $U$ is an open subspace of $X$ if $|U|$ is an open subspace of $|X|$ and $\mathcal{O}_{U}=\left.\mathcal{O}_{X}\right|_{|U|}$. A space $X$ is a closed subspace of $Y$ (denoted $X \hookrightarrow Y$ ) if there is a coherent sheaf of ideals $I \subset \mathcal{O}_{Y}$ such that $|X|=\operatorname{supp} \mathcal{O}_{Y} / I$ and $\mathcal{O}_{X}=\mathcal{O}_{Y} /\left.I\right|_{|X|}$. In this case $X$ is said to be defined by $I$. If $V$ and $W$ are closed subspaces of $Y$ defined by $I, J \subset \mathcal{O}_{Y}$, their intersection $V \cap W$ and union $V \cup W$ are the closed subspaces of $Y$ defined by $I+J$ and $I \cap J$, respectively. Intersections and unions of open subspaces are defined in the obvious way.

If $f: X \rightarrow Y$ is holomorphic and $W \hookrightarrow Y$ is a closed subspace defined by $J \subset \mathcal{O}_{Y}$, the sheaf of ideals generated by $f^{-1} J \subset \mathcal{O}_{X}$ is coherent, and the closed subspace of $X$ defined by it is the analytic inverse image $f^{-1} W$ of $W$. (Here, and sometimes in the sequel, we abuse notation by writing $f$ instead of $|f|$. Similarly, $X$ instead of $|X|$ may be written - the meanings should always be clear from context.) In particular, for $y \in Y, f^{-1}(y)$ is the fibre of $f$ over $y . f$ is an embedding if there is a closed subspace $X^{\prime} \hookrightarrow Y$ and an isomorphism $f^{\prime}: X \rightarrow X^{\prime}$ such that $f=i \circ f^{\prime}$, where $i: X^{\prime} \rightarrow Y$ is the inclusion mapping.

If $X$ and $Y$ are analytic spaces, their product $X \times Y$ is the analytic space $\left(|X| \times|Y|, \mathcal{O}_{X} \hat{\otimes}_{\mathbb{C}} \mathcal{O}_{Y}\right)$, which comes equipped with a pair of canonical holomorphic mappings (called projections) $\pi_{1}: X \times Y \rightarrow X$ and $\pi_{2}: X \times Y \rightarrow Y$, defined in the obvious way.

For an analytic space $X$, the analytic sheaf on $X$, whose stalk at $x \in X$ is the ideal of nilpotent elements of $\mathcal{O}_{X, x}$, is coherent, and the closed subspace it defines is the reduction $X_{\text {red }}$ of $X . X$ is reduced if $X=X_{\text {red }}$ and nonsingular if each $x \in X$ has an open neighbourhood isomorphic to an open subspace of $\mathbb{C}^{n}$. A reduced analytic space resembles a manifold with singularities, for example, $\left\{(x, y) \in \mathbb{C}^{2}: x y=0\right\}$; a nonsingular, paracomapact, analytic space is a complex manifold.

The usual definitions of vector bundles and sections thereof can be carried directly across from manifold theory: namely, a (rank $N$ holomorphic) vector bundle $E$ on an analytic space $X$ is an analytic space $E$ together with a holomorphic mapping $p$ : $E \rightarrow X$ such that for each $x \in X$, (a) $p^{-1}(x)$ has the structure of an $N$-dimensional 
complex vector space, and (b) there is an open neighbourhood $U$ of $x$ and an isomorphism $g: p^{-1}(U) \rightarrow U \times \mathbb{C}^{N}$ with $\pi_{1} \circ g=p$ and $\left.\pi_{2} \circ g\right|_{p^{-1}(x)}$ linear. A (holomorphic) section $s$ of $E$ over a subspace $V \subset X$ is a holomorphic mapping $s: V \rightarrow E$ such that $p \circ s=$ identity. If $\left(E_{1}, p_{1}\right)$ and $\left(E_{2}, p_{2}\right)$ are bundles on $X$, a morphism between them is a holomorphic map $h: E_{1} \rightarrow E_{2}$ such that $p_{2} \circ h=p_{1}$ and $\left.h\right|_{p_{1}^{-1}(x)}$ is linear, so with composition of morphisms defined in the obvious way, the set of vector bundles on $X$ forms a category. A bundle $E$ is trivial if it is isomorphic to $X \times \mathbb{C}^{N}$.

Just as for manifolds, the functor $E \mapsto \mathcal{O}(E):=$ sheaf of germs of holomorphic sections of $E$ defines an equivalence of the categories of vector bundles and locally free sheaves on $X$, so a bundle is determined by a system of invertible holomorphic matrices satisfying the cocycle conditions in exactly the same way as for manifolds. The set of rank $N$ vector bundles on $X$ modulo the equivalence relation of bundle isomorphism is thus in 1-1 correspondence with the pointed cohomology set $H^{1}\left(X, \mathrm{GL}\left(N, \mathcal{O}_{X}\right)\right)$; two bundles are isomorphic iff their images in $H^{1}\left(X, \operatorname{GL}\left(N, \mathcal{O}_{X}\right)\right)$ coincide. Sometimes the distinction between a bundle and its image in this cohomology set will be blurred in the sequel; that is, isomorphic bundles (and particularly those which are canonically isomorphic) may be regarded as the same object. Canonical isomorphisms will often be indicated by an equality, rather than isomorphism, symbol.

For a closed subspace $X$ of an analytic space $Y$, defined by $I \subset \mathcal{O}_{Y}$, the closed subspace $X^{(n)}$ of $Y$, defined by $I^{n+1}$, is called the $n$th formal neighbourhood of $X$ in $Y$. Thus $\left(X^{(n)}\right)_{\text {red }}=X_{\text {red }}$, and there is a filtration

$$
X=X^{(0)} \hookrightarrow X^{(1)} \hookrightarrow \cdots \hookrightarrow X^{(n)} \hookrightarrow \cdots \hookrightarrow Y
$$

with $X^{(n)} \hookrightarrow X^{(n+1)}$ defined by $I^{n+1} / I^{n+2} \subset \mathcal{O}_{X^{(n+1)}}$. (Here, and subsequently, the restriction symbol $\left.\right|_{|X|}$ is suppressed for any analytic sheaf supported on a closed subspace.) If $X$ and $Y$ are both nonsingular, $I^{n+1} / I^{n+2}$ is a locally free sheaf on $X$ : it is the sheaf of germs of sections of the $(n+1)$ th symmetric tensor power of the conormal bundle of $X$ in $Y$. In these circumstances, $\mathcal{O}_{X^{(n)}}$ has an obvious local representation in terms of truncated power series.

This section concludes on the same subject with which it began, namely the transport of sheaves under mappings.

If $f: X \rightarrow Y$ is a holomorphic mapping of analytic spaces and $\mathscr{S}$ is an analytic sheaf on $Y, f^{-1} \mathscr{S}$ is not, in general, an analytic sheaf on $X$. In order to remain within the analytic category, the analytic pull-back of $\mathscr{S}$ to $X$ is the analytic sheaf $f^{*} \mathscr{S}$ $:=\mathcal{O}_{X} \otimes_{f^{-1} \mathcal{O}_{Y}} f^{-1} \mathscr{S}$ on $X . f^{*}$ is a functor from the category of analytic sheaves on $Y$ to the category of analytic sheaves on $X$, and by right exactness of the tensor product, it preserves coherence. It also preserves local freeness: if $B$ is a bundle on $Y$, $f^{*} \mathcal{O}(B)=: \mathcal{O}\left(f^{*} B\right)$. Although $f^{*}$ is right exact, it is not, in general, an exact functor; when it is, $f$ is flat. Each projection on the product of two analytic spaces is flat (Fischer [11]).

Pushing sheaves in the opposite direction, suppose that $\mathscr{R}$ is an analytic sheaf on $X$. Then the direct images $f_{*}^{q} \mathscr{R}$ are all analytic sheaves on $Y$, but they are not 
necessarily coherent if $\mathscr{R}$ is. However, Grauert's celebrated theorem on direct images states that if $\mathscr{R}$ is coherent and $f$ is proper, then all the direct images are indeed coherent. In addition, one also has the Projection Formula

$$
f_{*}^{q}\left(\mathscr{R} \otimes \mathcal{O}\left(f^{*} B\right)\right)=\left(f_{*}^{q} \mathscr{R}\right) \otimes \mathcal{O}(B)
$$

for any bundle $B$ on $Y$, a fact frequently used in Part II. The functor $f_{*}^{q}$ does not necessarily preserve local freeness, but if $f$ is both flat and proper and $Y$ is reduced, then for a locally free sheaf $\mathscr{R}$ on $X$ such that $H^{q}\left(f^{-1}(y), \mathscr{R} \otimes \mathcal{O}_{f^{-1}(y)}\right)$ is of constant dimension for $y \in Y, f_{*}^{q} \mathscr{R}$ is a locally free sheaf on $Y$, and the corresponding bundle has fibre $H^{q}\left(f^{-1}(y), \mathscr{R} \otimes \mathcal{O}_{f^{-1}(y)}\right)$ at $y \in Y$ (Fischer [11]).

1.2. Differential forms on analytic spaces. Let $U$ be an open subset of $\mathbb{C}^{n}, I \subset \mathcal{O}_{U}$ a coherent sheaf of ideals, and $X$ the local model defined by $I$. Denote by $\mathcal{O}_{U} \cdot d I$ the sheaf of $\mathcal{O}_{U}$-modules generated by germs of the form $d f_{z}$ for $f_{z} \in I_{z}$ and $z \in U$, and set $\Omega_{X}^{1}:=\Omega_{U}^{1} / \mathcal{O}_{U} \cdot d I$, which is called the sheaf of germs of holomorphic 1-forms on $X$. The containment $I \cdot \Omega_{U}^{1} \subset \mathcal{O}_{U} \cdot d I$ gives rise to an exact sequence of sheaves of $\mathcal{O}_{U}$ modules, $I \stackrel{d}{\rightarrow} \Omega_{U}^{1} \otimes \mathcal{O}_{U} / I \rightarrow \Omega_{X}^{1} \rightarrow 0$, which implies that $\Omega_{X}^{1}$ is a coherent analytic sheaf on $X . \Omega_{X}^{1}$ is rarely locally free, however, this being the case iff $X$ is nonsingular (Fischer [11]).

If $Y$ is another local model and $f: X \rightarrow Y$ is holomorphic, there is an induced homomorphism $f^{*} \Omega_{Y}^{1} \rightarrow \Omega_{X}^{1}$ of analytic sheaves on $X$ denoted by $d f$. If $g: Y \rightarrow Z$ is holomorphic, the usual functorial equalities $d(g \circ f)=d f \circ f^{*} d g, d(\mathrm{id})=$ id hold (where id denotes the identity morphism), and it follows that the definitions of $\Omega_{X}^{1}$ and $d f$ are independent of the embedding $X \rightarrow U$ and, therefore, extend to arbitrary analytic spaces $X, Y$.

For a product of spaces $X, Y$, there is a canonical isomorphism $\Omega_{X \times Y}^{1} \simeq \pi_{1}^{*} \Omega_{X}^{1} \oplus$ $\pi_{2}^{*} \Omega_{Y}^{1}$.

Holomorphic $p$-forms $\Omega_{X}^{p}$ on an analytic space $X$ are defined by $\Omega_{X}^{p}:=\Lambda^{p} \Omega_{X}^{1}$ (with $\left.\Omega_{X}^{0}:=\mathcal{O}_{X}\right)$, and there is a well-defined differential $d: \Omega_{X}^{p} \rightarrow \Omega_{X}^{p+1}$ annihilating the constants and satisfying the usual Leibnitz rule. The complex of $\mathbb{C}$-modules $\Omega_{X}^{*}$ is called the holomorphic de Rham complex on $X$, but in general it is not a resolution of $\mathbb{C}$, even when $X_{\text {red }}$ is nonsingular (Grauert and Kerner [13]) or when $X$ is reduced (Reiffen [27]).

If $f: X \rightarrow Y$ is holomorphic, the cokernel of $d f: f^{*} \Omega_{Y}^{1} \rightarrow \Omega_{X}^{1}$ is denoted by $\Omega_{f}^{1}$, and $\Omega_{f}^{p}:=\Lambda^{p} \Omega_{f}^{1}$ is called the sheaf of germs of holomorphic relative $p$-forms on $X$. The induced differential $\Omega_{f}^{p} \rightarrow \Omega_{f}^{p+1}$ (differentiation along the fibres of $f$ ) is denoted by $d f$, and the complex of $f^{-1} \mathcal{O}_{Y}$-modules $\Omega_{f}$ is called the holomorphic relative de Rham complex on $X$. If $X$ and $Y$ are manifolds and $f$ is of maximal rank everywhere, the relative de Rham complex is a resolution of $f^{-1} \mathcal{O}_{Y}$. The question of its exactness or otherwise for other types of analytic spaces is considered in the next section.

With well-defined notions of differential forms and bundles on an analytic space, we can carry straight over from manifold theory the definition of connections on bundles: if $E$ is a bundle on a space $X$, let $\Omega_{X}^{p}(E):=\Omega_{X}^{p} \otimes_{\mathcal{O}_{X}} \mathcal{O}(E)$. A (holomorphic) connection $\nabla$ on $E$ is a $\mathbb{C}$-linear map $\nabla: \mathcal{O}(E) \rightarrow \Omega_{X}^{1}(E)$ satisfying the Leibnitz rule 
$\nabla(f s)=d f \otimes s+f \nabla s$ for $f \in \Gamma\left(U, \mathcal{O}_{X}\right), s \in \Gamma(U, \mathcal{O}(E))$ and $U \subset X$ open. Of course, $E$ need not admit a connection: if $M(N, \mathbb{C})$ denotes complex $N \times N$ matrices and $M(N, \mathscr{S}):=M(N, \mathbb{C}) \otimes_{\mathbb{C}} \mathscr{S}$ for a sheaf of $\mathbb{C}$-modules $\mathscr{S}$ on $X$, define $D$ : $\operatorname{GL}\left(N, \mathcal{O}_{X}\right) \rightarrow M\left(N, \Omega_{X}^{1}\right)$ by $D g:=g^{-1} d g$. Since $D(g h)=h^{-1}(D g) h+D h$, the corresponding map on cohomology is given by

$$
H^{1}\left(X, \mathrm{GL}\left(N, \mathcal{O}_{X}\right)\right) \ni E \mapsto D E \in H^{1}\left(X, \Omega_{X}^{1}(\text { End } E)\right)
$$

(where End $E:=\operatorname{Hom}(E, E)$ ). The class $D E$ is precisely the obstruction to defining a connection on $E$. (As forewarned in the last section), the bundle $E$ here is being identified with its equivalence class of isomorphic bundles. If $E$ is determined by a cocycle $\left(g_{i j}\right)$ with respect to some open covering $\left\{U_{i}\right\}_{i \in I}$ of $X$, a connection on $E$ as defined above is precisely a choice of splitting of the cocycle

$$
\left(g_{j i} d g_{i j}\right) \in Z^{1}\left(\left\{U_{i}\right\}, \Omega_{X}^{1}(\text { End } E)\right) .
$$

An isomorphism $\phi: E \stackrel{\sim}{\rightarrow} E^{\prime}$ determines a connection on $E^{\prime}$ given by $\nabla^{\prime}\left(s^{\prime}\right)=$ $\phi \nabla\left(\phi^{-1} s^{\prime}\right)$, which in terms of cocycles is just $D$ of the appropriate cochain added to the splitting of $\left(g_{j i} d g_{i j}\right)$. The map from cohomology with respect to an open covering into the direct limit is always injective on $H^{1}$ (for both abelian and nonabelian cohomology), so $D E \in H^{1}\left(X, \Omega_{X}^{1}\right.$ (End $\left.\left.E\right)\right)$ really is the obstruction to defining a connection on $E$. Regarding isomorphic bundles as the same object means that connections $\nabla_{1}, \nabla_{2}$ on a (representative) bundle $E$ must be regarded as equivalent if there is an isomorphism $\phi: E \rightarrow E$ such that $\nabla_{1}=\phi \circ \nabla_{2} \circ \phi^{-1}$; that is, $\nabla_{1}$ and $\nabla_{2}$ are gauge equivalent.)

If $\nabla_{1}$ and $\nabla_{2}$ are connections on $E_{1}$ and $E_{2}$, respectively, there are induced connections on $E_{1} \otimes E_{2}$ and $\operatorname{Hom}\left(E_{1}, E_{2}\right)$ characterized by

$$
\nabla\left(s_{1} \otimes s_{2}\right)=\left(\nabla_{1} s_{1}\right) \otimes s_{2}+s_{1} \otimes \nabla_{2} s_{2}
$$

and

$$
\nabla_{2}\left(\phi\left(s_{1}\right)\right)=\nabla(\phi)\left(s_{1}\right)+\phi\left(\nabla_{1} s_{1}\right)
$$

for local sections $s_{i}$ of $E_{i}$ and $\phi$ of $\operatorname{Hom}\left(E_{1}, E_{2}\right)$. If $\nabla_{1}$ and $\nabla_{2}$ are two connections on $E$, their difference defines an $\mathcal{O}_{X}$-module homomorphism $\mathcal{O}(E) \rightarrow \Omega_{X}^{1}(E)$, and this homomorphism is realized as the section $\nabla$ (id) $\in \Gamma\left(X, \Omega_{X}^{1}(\right.$ End $\left.E)\right)$, where $\nabla$ is the connection induced on End $E$ by $\nabla_{1}$ and $\nabla_{2}$ together. If $g: X \rightarrow Y$ is holomorphic and $B$ is a bundle on $Y$, a connection on $B$ induces a connection $g^{*} B$ characterized by $\nabla\left(g^{*} s\right)=d g \circ g^{*} \nabla s$ for a local section $s$ of $E$. Note that, as a special case of this, a connection on $B$ induces a connection $\left.B\right|_{X}$ if $X \hookrightarrow Y$ is a closed subspace.

If $E$ is a bundle on an analytic space $X$ with connection $\nabla$, a local section $s$ of $E$ is covariantly constant if $\nabla s=0$. When morphisms are defined to be bundle morphisms covariantly constant with respect to the induced connection (i.e., $\phi: E_{1} \rightarrow E_{2}$ must satisfy $\nabla_{2} \circ \phi=\phi \circ \nabla_{1}$ ), the collection of bundles with connection on an analytic space forms a category.

In general, a bundle $E$ with connection $\nabla$ has no covariantly constant local sections: there are induced $\mathbb{C}$-linear maps $\nabla: \Omega_{X}^{p}(E) \rightarrow \Omega_{X}^{p+1}(E)$, defined by

$$
\nabla(h \otimes s):=d h \otimes s+(-1)^{p} h \wedge \nabla s
$$


for a $p$-form $h$ and section $s$, but the composition $\nabla^{2}: \mathcal{O}(E) \rightarrow \Omega_{X}^{2}(E)$ is not usually zero. It is, however, an $\mathcal{O}_{X}$-module homomorphism, and the section $\mathbf{F}=\mathbf{F}(\nabla) \in$ $\Gamma\left(X, \Omega_{X}^{2}(\right.$ End $\left.E)\right)$ defined by $\nabla^{2}$ is called the curvature of $\nabla$. There is an identity $\nabla \mathbf{F}=0$, called the Bianchi identity, where $\nabla$ denotes the induced connection on End $E$. The curvature depends functorially on the bundle and connection in the obvious way, i.e.,

$$
\mathbf{F}\left(\nabla_{1} \otimes \nabla_{2}\right)=\mathbf{F}\left(\nabla_{1}\right) \otimes \mathrm{id}+\mathrm{id} \otimes \mathbf{F}\left(\nabla_{2}\right),
$$

etc.

A connection $\nabla$ on $E$ is flat if $\mathbf{F}(\nabla)=0$. If $X$ is a manifold and $\nabla$ is flat, then, by the Frobenius theorem, $(E, \nabla) \simeq\left(E^{\prime}, d\right)$, where $E^{\prime} \in H^{1}(X, \operatorname{GL}(N, \mathbb{C}))$. If $X$ is connected and $x_{0} \in X$,

$$
H^{1}(X, \mathrm{GL}(N, \mathbb{C})) \simeq \operatorname{Hom}\left(\pi_{1}\left(X, x_{0}\right), \operatorname{GL}(N, \mathbb{C})\right)
$$

(Gunning [15]), so if $X$ is simply connected also, the bundle $E$ is canonically trivialized by its covariantly constant sections. Stated precisely, in these circumstances, $V:=\{s \in \Gamma(X, \mathcal{O}(E)): \nabla s=0\}$ is an $N$-dimensional complex vector space, and the canonical map $\left(\mathcal{O}_{X} \otimes_{\mathrm{C}} V, d\right) \rightarrow(\mathcal{O}(E), \nabla)$ is an isomorphism.

In complete analogy, if $f: X \rightarrow Y$ is a holomorphic mapping of analytic spaces and $E$ is a bundle on $X$, a relative connection on $E$ is a $\mathbb{C}$-linear map $\nabla_{f}$ : $\mathcal{O}(E) \rightarrow \Omega_{f}^{1}(E)$ satisfying $\nabla_{f}(h s)=d_{f} h \otimes s+h \nabla_{f} s$ for a function $h$ and a section $s$. These remarks and definitions concerning connections can now be repeated with obvious modifications, replacing $\nabla$ by $\nabla_{f}$ and $d$ by $d_{f}$ throughout. It should be additionally noted that the obstruction $D_{f} E$ to the existence of a relative connection on $E$ coincides with the image of $D E$ in $H^{1}\left(X, \Omega_{f}^{1}(\right.$ End $\left.E)\right)$, and a connection on $E$ induces a relative connection on $E$. In the particular case when $E=f^{*} B$ for some bundle $B$ on $Y$, the functorial identity $D\left(f^{*} B\right)=d f \circ f^{*} D B$ implies $D_{f} E=0$ automatically. Indeed, in these circumstances there is a canonical relative connection on $E$ denoted by $d_{f}$ and characterized by $d_{f}\left(f^{*} s\right)=0$ for any local section $s$ of $B . d_{f}$ is flat, so the sequence $\cdots \rightarrow \Omega_{f}^{p}\left(f^{*} B\right) \rightarrow \Omega_{f}^{p+1}\left(f^{*} B\right) \rightarrow \cdots$ is a complex. Since $f^{-1} \mathcal{O}(B)$ is a locally free sheaf of $f^{-1} \mathcal{O}_{Y}$-modules, this complex is a resolution of $f^{-1} \mathcal{O}(B)$ when the relative de Rham complex is exact.

1.3. The relative de Rham complex on formal neighbourhoods. The local definition of 1-forms in the previous section implies that for arbitrary $X, Y, \Omega_{X}^{1}=\Omega_{Y}^{1} / \mathcal{O}_{Y} \cdot d I$ where $I \subset \mathcal{O}_{Y}$ defines $X \hookrightarrow Y$ and $\mathcal{O}_{Y} \cdot d I$ has the same meaning as before. When $I$ is replaced by $I^{n+1}$, the containments $I^{n+1} \cdot \Omega_{Y}^{1} \subset \mathcal{O}_{Y} \cdot d I^{n+1} \subset I^{n} \cdot \Omega_{Y}^{1}$ give rise to epimorphisms $\Omega_{Y}^{1} \otimes \mathcal{O}_{X^{(n)}} \rightarrow \Omega_{X^{(n)}}^{1}$ and $\Omega_{X^{(n)}}^{1} \rightarrow \Omega_{Y}^{1} \otimes \mathcal{O}_{X^{(n-1)}}$, the kernels of which are coherent analytic sheaves on $X$. More generally, since $\Omega_{X^{(n)}}^{p}=\Omega_{Y}^{p} / \Omega_{Y}^{p-1} \wedge d I$, there are exact sequences

$$
\Omega_{Y}^{p-1} \otimes I^{n+1} / I^{n+2} \rightarrow \Omega_{Y}^{p} \otimes \mathcal{O}_{X^{(n)}} \rightarrow \Omega_{X^{(n)}}^{p} \rightarrow 0,
$$

and

$$
\Omega p \otimes I^{n} / I^{n+1} \rightarrow \Omega_{X^{(n)}}^{p} \rightarrow \Omega_{Y}^{p} \otimes \mathcal{O}_{X^{(n-1)}} \rightarrow 0
$$


Let $S_{Y^{(n)}}^{p}$ denote the kernel of the map $\Omega_{X^{(n+1)}}^{p} \rightarrow \Omega_{X^{(n)}}^{p}$, so, with the induced differentials on $S_{X^{(n)}}^{p}$, there is an exact sequence of complexes

$$
0 \rightarrow S_{X^{(n)}}^{\cdot} \rightarrow \Omega_{X^{(n+1)}}^{\cdot} \rightarrow \Omega_{X^{(n)}}^{\cdot \rightarrow 0}
$$

Note that $S_{X^{(n)}}^{p}$ is an anlytic sheaf on $X^{(1)}$, but not on $X$.

Now suppose $X$ and $Y$ are nonsingular. Then $0 \rightarrow I / I^{2} \rightarrow \Omega_{Y}^{1} \otimes \mathcal{O}_{X} \rightarrow \Omega_{X}^{1} \rightarrow 0$ is an exact sequence of locally free sheaves on $X$, and, therefore, its $q$ th exterior power

$$
0 \rightarrow I^{q} / I^{q+1} \rightarrow \Omega_{Y}^{1} \otimes I^{q-1} / I^{q} \rightarrow \cdots \rightarrow \Omega_{Y}^{q-1} \otimes I / I^{2} \rightarrow \Omega_{Y}^{q} \otimes \mathcal{O}_{X} \rightarrow \Omega_{X}^{q} \rightarrow 0
$$

is exact for all $q$ (hitherto (1.3.4) has only been complex). Using (1.3.1), (1.3.2) and (1.3.4), it is straightforward to deduce that there is an exact sequence of analytic sheaves on $X^{(1)}$,

$$
0 \rightarrow R_{X^{(n)}}^{p+1} \rightarrow S_{X^{(n)}}^{p} \rightarrow R_{X^{(n)}}^{p} \rightarrow 0
$$

where

$R_{X^{(n)}}^{p}:=\operatorname{ker} \Omega_{Y}^{p} \otimes \mathcal{O}_{X^{(n)}} \rightarrow \Omega_{X^{(n)}}^{p}=\operatorname{coker} \Omega_{Y}^{p-2} \otimes I^{n+2} / I^{n+3} \rightarrow \Omega_{Y}^{p^{-1}} \otimes I^{n+1} / I^{n+2}$.

The composition $R_{X^{(n)}}^{p+1} \rightarrow S_{X^{(n)}}^{p} \stackrel{d}{\rightarrow} S_{X^{(n)}}^{p+1} \rightarrow R_{X^{(n)}}^{p+1}$ is the identity (so $d$ induces a canonical splitting $S_{X^{(n)}}^{p} \simeq R_{X^{(n)}}^{p+1} \oplus R_{X^{(n)}}^{p}$ as a sheaf of $\mathbb{C}$-modules), and one immediately obtains

Proposition A. In the above circumstances, $H^{*}\left(H^{q}\left(U, S_{X^{(n)}}\right)\right)=0$ for all $q$ and any open $U \subset X$.

Since $\Omega_{X}$ is a resolution of $\mathbb{C}$ by the holomorphic de Rham theorem, induction applied to (1.3.3) gives

Corollary A. For a submanifold $X$ of a manifold $Y, \Omega_{X^{(n)}}$ is a resolution of $\mathbb{C}$ for all $n$.

Now suppose $V$ is another complex manifold and $F: Y \rightarrow V, f: X \rightarrow V$ are both surjective holomorphic mappings of maximal rank such that $f=\left.F\right|_{X}$. Then $f^{(n)}:=\left.F\right|_{X^{(n)}}$ is a surjective (indeed, flat) holomorphic mapping of $X^{(n)}$ onto $V$, and all of the preceding analysis can be repeated with the formal substitutions $\Omega_{Y}^{p} \mapsto \Omega_{F}^{p}$, $\Omega_{X^{(n)}}^{p} \mapsto \Omega_{f^{(n)}}^{p^{\prime}}, d($ on $Y) \mapsto d_{F}, d\left(\right.$ on $\left.X^{(n)}\right) \mapsto d_{f^{(n)}}, S_{X^{(n)}}^{p} \mapsto S_{f^{(n)}}^{p}$ and $R_{X^{(n)}}^{p} \mapsto R_{f^{(n)}}^{p}$. In other words, the above analysis can be repeated on $f^{-1}(v) \hookrightarrow F^{-1}(v)$ parametrized by $v \in V$. Hence,

Proposition B. In the above circumstances $\Omega_{f^{(n)}}$ is a resolution of $f^{-1} \mathcal{O}_{V}$ for all $n$.

Continuing with this process of generalization, suppose now that $Y$ is a submanifold of a manifold $Z$ defined by $J \subset \mathcal{O}_{Z}$. If $K \subset \mathcal{O}_{Z}$ denotes the coherent sheaf of ideals defining $X$ as a submanifold of $Z$, then $J \subset K$ and $I=K / J \subset \mathcal{O}_{Z} / J=\mathcal{O}_{Y}$. Moreover, one has the exact sequence

$$
0 \rightarrow J / J^{2} \otimes \mathcal{O}_{X} \rightarrow K / K^{2} \rightarrow I / I^{2} \rightarrow 0
$$

of conormal bundles on $X$. 
$X$ now has two sets of formal neighbourhoods, namely those coming from each of the embeddings $X \hookrightarrow Y$ and $X \hookrightarrow Z$. To distinguish them let $X^{[n]}$ denote the $n$th formal neighbourhood of $X$ in $Z$, and $X^{(n)}$ its $n$th formal neighbourhood in $Y$, as before. Since

$$
I^{n+1}=(K / J)^{n+1}=K^{n+1} / K^{n+1} \cap J=\left(K^{n+1}+J\right) / J \subset \mathcal{O}_{Z} / J
$$

$X^{(n)}$ is defined by $K^{n+1}+J$ as a closed subspace of $Z$. Thus $X^{(n)}$ is a closed subspace of $X^{[n]}$ defined by $J / K^{n+1} \cap J \subset \mathcal{O}_{X^{[n]}}$. Now let $X^{(n, m)}$ denote the $m$ th formal neighbourhood of $X^{(n)}$ in $X^{[n]}$; it is defined by

$$
J^{m+1} / K^{n+1} \cap J^{m+1}=\left(J^{m+1}+K^{n+1}\right) / K^{n+1} \subset \mathcal{O}_{X^{[n]}},
$$

so $X^{(n, m)}=X^{[n]} \cap Y^{(m)}$. In this case the filtration (1.1.1) is finite:

$$
X^{(n)}=X^{(n, 0)} \hookrightarrow X^{(n, 1)} \hookrightarrow \cdots \hookrightarrow X^{(n, n)}=X^{[n]} .
$$

Note that $K^{n+1} \cap J^{n+1}=K^{n-m} \cdot J^{m+1}$ for $m \leqslant n$, so

$$
\left(J^{m+1}+K^{n+1}\right) / K^{n+1}=J^{n+1} / K^{n-m} J^{m+1}=J^{n+1} \otimes \mathcal{O}_{X^{[n-m-1]}} .
$$

From (1.3.1) one has the exact sequence

$$
\Omega_{Z}^{p-1} \otimes \frac{K^{n+1}+J^{m+1}}{K^{n+2}+J^{m+2}} \rightarrow \Omega_{Z}^{p} \otimes \mathcal{O}_{X^{(n, m)}} \rightarrow \Omega_{X^{(n, m)}}^{p} \rightarrow 0,
$$

which can be extended on the left to a complex

$$
\cdots \rightarrow \Omega_{Z}^{p-k} \otimes \frac{K^{n+k}+J^{m+k}}{K^{n+k+1}+J^{m+k+1}} \rightarrow \Omega_{Z}^{p-k+1} \otimes \frac{K^{n+k-1}+J^{m+k-1}}{K^{n+k}+J^{m+k}} \rightarrow \cdots .
$$

There is an exact sequence

$$
0 \rightarrow \frac{J^{r+1}}{J^{r+2}} \otimes \mathcal{O}_{X^{(q-r-1)}} \rightarrow \frac{K^{q}+J^{r+1}}{K^{q+1}+J^{r+2}} \rightarrow \frac{K^{q}+J^{r}}{K^{q+1}+J^{r+1}} \rightarrow \frac{J^{r}}{J^{r+1}} \otimes \mathcal{O}_{X^{(q-r-1)}} \rightarrow 0 .
$$

It follows easily, by using induction on $m$ together with the exactness of the exterior powers of $0 \rightarrow J / J^{2} \rightarrow \Omega_{Z}^{1} \otimes \mathcal{O}_{Y} \rightarrow \Omega_{Y}^{1} \rightarrow 0$, that the sequence obtained by combining (1.3.7) and (1.3.8) is exact. This fact, together with some simple homological algebra, enables one to deduce that the kernel $S_{X^{(n, m)}}^{p}$ of the epimorphism $\Omega_{X^{(n, m+1)}}^{p} \rightarrow$ $\Omega_{X^{(n, m)}}^{p}$ fits into an exact sequence analogous to (1.3.5):

$$
0 \rightarrow R_{Y^{(m)}}^{p+1} \otimes \mathcal{O}_{X^{(n-m-1)}} \rightarrow S_{X^{(n, m)}}^{p} \rightarrow R_{Y^{(m)}}^{p} \otimes \mathcal{O}_{X^{(n-m-1)}} \rightarrow 0 .
$$

The same conclusions as for Proposition A follow, giving

Proposition C. In the above circumstances $\Omega_{X^{(n, m)}}$ is a resolution of $\mathbb{C}$ for all $n, m$.

This proposition will not be subsequently used, but it was included for the sake of completeness. The important result needed in Part II is the appropriate generalization of Proposition B. The set-up for this is indicated by the following diagram:

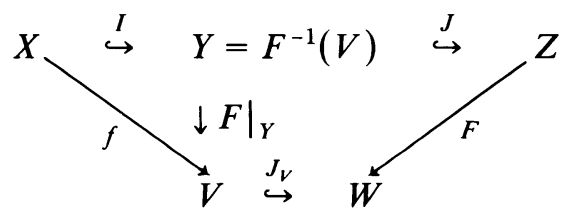


Here $F: Z \rightarrow W$ is again surjective and of maximal rank, and $J_{V} \subset \mathcal{O}_{W}$ defines $V$. Thus $J \simeq F^{*} J_{V}$ and $\left.\Omega_{F}^{1}\right|_{Y}=\Omega_{F \mid Y}^{1}$.

The restriction of $F$ to $X^{(n, m)}$ defines a surjective holomorphic mapping $f^{(n, m)}$ : $X^{(n, m)} \rightarrow V^{(m)}$. The exact sequence (1.3.7) has as its relative analogue the exact sequence

$$
\Omega_{F}^{p-1} \otimes \frac{K^{n+1}+J^{m+1}}{K^{n+2}+J^{m+1}} \rightarrow \Omega_{F}^{p} \otimes \mathcal{O}_{X^{(n, m)}} \rightarrow \Omega_{f^{(n, m)}}^{p} \rightarrow 0,
$$

and again there is an extension of this sequence to a complex

$$
\cdots \rightarrow \Omega_{F}^{p-k} \otimes \frac{K^{n+k}+J^{m+1}}{K^{n+k+1}+J^{m+1}} \rightarrow \Omega_{F}^{p-k+1} \otimes \frac{K^{n+k-1}+J^{m+1}}{K^{n+k}+J^{m+1}} \rightarrow \cdots .
$$

The sheaf $\left(K^{q}+J^{r}\right) /\left(K^{q+1}+J^{r}\right)$ is the (locally free) analytic sheaf on $X$ which is the cokernel of the homomorphism $K^{q-r} / K^{q-r+1} \otimes J^{r} / J^{r+1} \rightarrow K^{q} / K^{q+1}$. Some straightforward multilinear algebra shows that the cohomology of the complex

$$
\Omega_{F}^{s-1} \otimes \frac{K^{q+1}+J^{r}}{K^{q+2}+J^{r}} \rightarrow \Omega_{F}^{s} \otimes \frac{K^{q}+J^{r}}{K^{q+1}+J^{r}} \rightarrow \Omega_{F}^{s+1} \otimes \frac{K^{q-1}+J^{r}}{K^{q}+J^{r}}
$$

is 0 if $r \leqslant q$ and $\Omega_{f}^{s} \otimes J^{q} / J^{q+1}$ if $r>q$. Since one can assume without loss of generality that $m \leqslant n$, it follows that the sequence obtained by combining (1.3.10) and (1.3.11) is exact, and this leads easily to the identification of the kernel of $\Omega_{f^{(n, m+1)}}^{p} \rightarrow \Omega_{f^{(n, m)}}^{p}$ as $\Omega_{f^{(n-m-1)}}^{p} \otimes J^{m+1} / J^{m+2}$. The induced differential on $\Omega_{f^{(n-m-1)}} \otimes$ $J^{m+1} / J^{m+2}$ is just $d_{f}$ on $\Omega_{f^{(n-m-1)}}^{\cdot}\left(F^{*} J_{V}^{m+1} / J_{V}^{m+2}\right)$, so induction on $m$ and Proposition $\mathrm{B}$ together give

Proposition D. In the above circumstances the exact sequence of complexes

$$
0 \rightarrow \Omega_{f^{(n-m-1)}} \otimes J^{m+1} / J^{m+2} \rightarrow \Omega_{f^{(n, m+1)}} \rightarrow \Omega_{f^{(n, m)}}^{\cdot \rightarrow 0}
$$

is a resolution of the topological inverse image of

$$
0 \rightarrow J_{V}^{m+1} / J_{V}^{m+2} \rightarrow \mathcal{O}_{V^{(m+1)}} \rightarrow \mathcal{O}_{V^{(m)}} \rightarrow 0 .
$$

In fact, for any $q \geqslant 0$, Proposition $\mathrm{D}$ and induction together allow one to conclude that there is an exact sequence of complexes

$$
0 \rightarrow \Omega_{f^{(n-m-1 . q-1)}}^{\cdot} \otimes J^{m+1} / J^{m+q+1} \rightarrow \Omega_{f^{(n, m+q)}}^{\cdot \rightarrow} \Omega_{f^{(n, m)}} \rightarrow 0
$$

resolving the topological inverse of

$$
0 \rightarrow J_{V}^{m+1} / J_{V}^{m+q+1} \rightarrow \mathcal{O}_{V^{(m+q)}} \rightarrow \mathcal{O}_{V^{(m)}} \rightarrow 0 .
$$

1.4. Inverting pull-back functors. So far, three main categories of analytic objects on an analytic space have been encountered: cohomology with coefficients in a coherent analytic sheaf, holomorphic vector bundles, and vector bundles with (relative) connection. Each of these categories admits a nice pull-back functor induced by a holomorphic mapping, and the general problem to be considered in this (and the next) section is that of determining when such a functor is 1-1 and/or surjective. In complete generality, there is, of course, little of practical value which can be said about this problem, but, by restricting oneself to the relatively uncomplicated situation considered in the last section, useful results can be obtained. For 
this purpose all the various spaces, maps, and notations of $\S 1.3$ will be carried over directly to this section.

For a bundle $B^{(m)}$ on $V^{(m)}$, a necessary condition for some element of $H^{p}\left(X^{(n, m)}, \mathcal{O}\left(f^{(n, m)^{*}} B^{(m)}\right)\right)$ to be a pull-back from $V^{(m)}$ is that it lie in the image of

$$
H^{p}\left(X^{(n, m)}, f^{-1} \mathcal{O}\left(B^{(m)}\right)\right) \rightarrow H^{p}\left(X^{(n, m)}, \mathcal{O}\left(f^{(n, m)^{*}} B^{(m)}\right)\right) ;
$$

this condition is described by chasing the chosen element through the cohomology of the resolution $\Omega_{f^{(n, m)}}\left(f^{(n, m)^{*}} B^{(m)}\right)$ of $f^{-1} \mathcal{O}\left(B^{(m)}\right)$, a process not worth describing for the illumination it provides. Getting from $H^{p}\left(X^{(n, m)}, f^{-1} \mathcal{O}\left(B^{(m)}\right)\right)$ down to $H^{p}\left(V^{(m)}, \mathcal{O}\left(B^{(m)}\right)\right)$ is a purely topological problem, and sufficient conditions for this inversion are given in

Proposition E. If, for any $v \in V, f^{-1}(v)$ is connected and satisfies $H^{q}\left(f^{-1}(v), \mathbb{C}\right)$ $=0$ for $q=1, \ldots, N$, the canonical map $H^{q}\left(V, \mathcal{O}\left(B^{(m)}\right)\right) \rightarrow H^{q}\left(X, f^{-1} \mathcal{O}\left(B^{(m)}\right)\right)$ is an isomorphism for $q=0,1, \ldots, N$ and a monomorphism for $q=N+1$.

Proof. The case $m=0$ is proved in Buchdahl [5]. The general case follows immediately by induction and the Five Lemma applied to the pair of long cohomology sequences resulting from

$$
0 \rightarrow \mathcal{O}(B) \otimes J_{V}^{q+1} / J_{V}^{q+2} \rightarrow \mathcal{O}\left(B^{(q+1)}\right) \rightarrow \mathcal{O}\left(B^{(q)}\right) \rightarrow 0
$$

and its topological inverse image, where $B^{(q)}:=\left.B^{(m)}\right|_{V^{(q)}}$ for $q \leqslant m$.

The case of bundles is, in a sense, less complicated, since one is dealing only with $H^{1}$, rather than $H^{q}$, cohomology, albeit nonabelian. In contrast to the abelian cohomology case, for bundles there is a succinct analytic description of the condition that an object be a pull-back, and this is given in

Proposition F. Suppose the fibres of $f$ are all connected and simply connected. Then for every bundle $E^{(n, m)}$ on $X^{(n, m)}$ with flat relative connection $\nabla_{f^{(n, m)}}: \mathcal{O}\left(E^{(n, m)}\right) \rightarrow$ $\Omega_{f^{(n, m)}}^{1}\left(E^{(n, m)}\right)$, there is a bundle $B^{(m)}$ on $V^{(m)}$, which is unique up to isomorphism, such that

$$
\left(E^{(n, m)}, \nabla_{f^{(n, m)}}\right) \simeq\left(f^{\left.(n, m)^{*} B^{(m)}, d_{f^{(n, m)}}\right) .}\right.
$$

Proof. The conclusion can be restated as saying that $f_{*} \operatorname{ker} \nabla_{f^{(n, m)}}=: \mathcal{O}\left(B^{(m)}\right)$ is a locally free analytic sheaf on $V^{(m)}$ such that the canonical map $f^{(n, m)^{*}} \mathcal{O}\left(B^{(m)}\right) \rightarrow$ $\mathcal{O}\left(E^{(n, m)}\right)$ is an isomorphism.

The uniqueness statement follows from the connectivity of the fibres of $f$ and from Propositions D and E.

The proof of existence is by induction, first on $n$ with $m=0$, and subsequently on $m$ with $n$ fixed. If $U \subset V$ is sufficiently small, the inverse function theorem implies that there exists a submanifold $U^{\prime} \subset f^{-1}(U)$ such that $\left.f\right|_{U^{\prime}}: U^{\prime} \rightarrow U$ is an isomorphism. $\nabla_{f}$ on $E:=E^{(0,0)}$ induces a flat connection on $\left.E\right|_{f^{-1}(u)}$ for each $u \in U$, giving a canonical trivialization of the bundle over this connected and simply-connected manifold. A map $s:\left.f^{-1}(U) \rightarrow E\right|_{f^{-1}(U)}$ is thus uniquely determined by prescribing an initial section of $\left.E\right|_{U^{\prime}}$ and covariantly propagating along the fibres of $f$. Using the Frobenius and Inverse Function theorems, we easily see that $s$ is a holomorphic 
section of $E$, and therefore a basis of sections of $\left.E\right|_{U^{\prime}}$ extends to a basis of covariantly-constant sections of $\left.E\right|_{f^{-1}(U)}$. Thus, $f_{*} \operatorname{ker} \nabla_{f}=: \mathcal{O}(B)$ is a locally free analytic sheaf on $V$ and, by construction, $\left(f^{*} B, d_{f}\right) \simeq\left(E, \nabla_{f}\right)$.

Now suppose $n+1>m=0$. An inspection of the analysis preceding Proposition $B$ reveals that it remains perfectly valid if all the sheaves appearing in it are replaced by their tensor products with $\mathcal{O}\left(E^{(n+1)}\right)$ and the differentials are replaced by differentials induced by $\nabla_{f^{(n+1)}}$. Thus, the conclusions of that analysis remain valid, which implies that the restriction map $\operatorname{ker} \nabla_{f^{(n+1)}} \rightarrow \operatorname{ker} \nabla_{f^{(n)}}$ is an isomorphism. Thus $f_{*} \operatorname{ker} \nabla_{f^{(n+1)}}=f_{*} \operatorname{ker} \nabla_{f^{(n)}}=\mathcal{O}(B)$ is locally free, and, again,

$$
\left(f^{(n+1)^{*}} B, d_{f^{(n+1)}}\right) \simeq\left(E^{(n+1)}, \nabla_{f^{(n+1)}}\right) .
$$

Finally, suppose $n \geqslant m+1>0$. This time the analysis preceding Proposition D can be tensored through by $\mathcal{O}\left(E^{(n, m+1)}\right)$ by replacing $d_{f^{(n, m+1)}}$ by $\nabla_{f^{(n, m+1)}}$. In $(1.3 .12) \otimes \mathcal{O}\left(E^{(n, m+1)}\right)$, the (flat) relative connection induced on

$$
\mathcal{O}\left(E^{(n-m-1)}\right) \otimes J^{m+1} / J^{m+2}
$$

is the same as that induced by $\nabla_{f^{(n, m+1)}}$ on

$$
E^{(n-m-1)} \quad\left(:=\left.E^{(n, m+1)}\right|_{X^{(n-m-1)}}\right)
$$

and $d_{f^{(n-m-1)}}$ on $\mathcal{O}_{X^{(n-m-1)}} \otimes J^{m+1} / J^{m+2}=f^{(n-m-1)^{*}} J_{V}^{m+1} / J_{V}^{m+2}$. It follows that the sequence

$$
0 \rightarrow f^{-1} \mathcal{O}(B) \otimes J_{V}^{m+1} / J_{V}^{m+2} \rightarrow \operatorname{ker} \nabla_{f^{(n, m+1)}} \rightarrow f^{-1} \mathcal{O}\left(B^{(m)}\right) \rightarrow 0
$$

is exact, and, applying $f_{*}$ and using Proposition $\mathrm{E}$, one obtains the exact sequence

$$
0 \rightarrow \mathcal{O}(B) \otimes J_{V}^{m+1} / J_{V}^{m+2} \rightarrow f_{*} \operatorname{ker} \nabla_{f^{(n, m+1)}} \rightarrow \mathcal{O}\left(B^{(m)}\right) \rightarrow 0
$$

of analytic sheaves on $V^{(m+1)}$. This sequence, together with Nakayama's Lemma, is sufficient to show that $f_{*} \operatorname{ker} \nabla_{f^{(n, m+1)}}$ is a locally free analytic sheaf on $V^{(m)}$, thereby defining $B^{(m+1)}$. By construction, the canonical map

$$
\left(f^{(n, m+1)^{*}} B^{(m+1)}, d_{f^{(n, m+1)}}\right) \rightarrow\left(E^{(n, m+1)}, \nabla_{f^{(n, m+1)}}\right)
$$

is an isomorphism. This completes the proof.

In the language of abstract nonsense, the assignment $B^{(m)} \mapsto\left(f^{(n, m)^{*}} B^{(m)}, d_{f^{(n, m)}}\right)$ defines a functor from the category of bundles on $V^{(m)}$ to the category of bundles on $X^{(n, m)}$ with flat relative connection. When $f$ has connected fibres, the functor is injective; when the fibres of $f$ are both connected and simply connected, the functor defines an equivalence of categories.

Finally, we have the case of connections, which is included here more for completeness than for application in Part II.

If $\left(E^{(n, m)}, \nabla^{(n, m)}\right)$ is a bundle with connection in $X^{(n, m)}$, a necessary condition that it be the pull-back of a bundle with connection on $V^{(m)}$ is that the curvature $\mathbf{F}^{(n, m)}=\mathbf{F}\left(\nabla^{(n, m)}\right)$ lie in the image of

$$
H^{0}\left(X^{(n, m)}, f^{(n, m)^{*}} \Omega_{V^{(n)}}^{2}\left(\text { End } E^{(n, m)}\right)\right) \rightarrow H^{0}\left(X^{(n, m)}, \Omega_{X^{(n, m)}}^{2}\left(\text { End } E^{(n, m)}\right)\right) .
$$

If this is the case, then the relative connection $\nabla_{f^{(n, m)}}$ induced by $\nabla^{(n, m)}$ is certainly flat, so if $f$ has connected and simply-connected fibres, Proposition $F$ gives an 
(essentially unique) bundle $B^{(m)}$ on $V^{(m)}$ such that

$$
\left(f^{(n, m)^{*}} B^{(m)}, d_{f^{(n, m)}}\right) \simeq\left(E^{(n, m)}, \nabla_{f^{(n, m)}}\right),
$$

and the problem is reduced to pushing $\nabla^{(n, m)}$ down to a connection on $B^{(m)}$.

When $n=m=0$, the injectivity of $d f$ implies that for any local section $s$ of $B$ $\left(=B^{(0)}\right), \nabla f^{*} s$ is the image of a unique section of $f^{*} \Omega_{V}^{1}(B)$, which, by the restriction on $\mathbf{F}(\nabla)$, is covariantly constant with respect to $d_{f}$. Thus one obtains a map $f^{-1} \mathcal{O}(B) \rightarrow f^{-1} \Omega_{V}^{1}(B)$ satisfying the appropriate Leibnitz rule, and since $f$ has connected fibres, this pushes down under $f_{*}$ to a (patently unique) connection on $B$ which induces $\nabla$ on $E$.

The general case is analogous: we must show that $d f^{(n, m)}$ is injective and the complex $\Omega_{f^{(n, m)}}^{\cdot} \otimes f^{(n, m)^{*}} \Omega_{V^{(m)}}^{1}$ is a resolution of $f^{-1} \Omega_{V^{(m)}}^{1}$ (even though $\Omega_{V^{(m)}}^{1}$ is not locally free). This is proved by showing that there is an exact sequence of complexes

$$
0 \rightarrow \Omega_{f^{(n-m)}} \otimes J^{m+1} / J^{m+2} \rightarrow \Omega_{f^{(n, m)}}^{\cdot} \otimes F^{*} \Omega_{W}^{1} \rightarrow \Omega_{f^{(n, m)}}^{\dot{2}} \otimes f^{(n, m)^{*}} \Omega_{V^{(m)}}^{1} \rightarrow 0,
$$

which is obtained by using the same types of arguments as in $\S 1.3$. Details are omitted, but one can state

Proposition G. Suppose $f$ has connected fibres and $B^{(m)}$ is a bundle on $V^{(m)}$ such that $f^{(n, m)^{*}} B^{(m)}$ admits a connection $\nabla^{(n, m)}$. Then if $\mathbf{F}\left(\nabla^{(n, m)}\right)$ lies in the image of

$$
H^{0}\left(X^{(n, m)}, f^{(n, m)^{*}} \Omega_{V^{(m)}}^{2}\left(\text { End } B^{(m)}\right)\right) \rightarrow H^{0}\left(X^{(n, m)}, \Omega_{X^{(n, m)}}^{2}\left(f^{(n, m)^{*}} \text { End } B^{(m)}\right)\right),
$$

there is a unique connection $\nabla^{(m)}$ on $B^{(m)}$ inducing $\nabla^{(n, m)}$ on $f^{(n, m)^{*}} B^{(m)}$.

There are, of course, obvious generalizations of the proposition for the case of relative connections.

1.5. The extension problem. In this section the question of the invertibility of an analytic pull-back functor is further considered, but this time at the other end of the spectrum of holomorphic mappings - namely, the pull-back functor induced by the inclusion mapping of one space as a closed subspace of another. The generic term for a problem of this type is the extension problem, which consists of determining when a certain analytic object on a closed subspace $X$ of an analytic space $Y$ is the restriction of a corresponding analytic object on $Y$, and to what extent any such extension of the object is unique. The underlying assumption is that the analytic cohomology of $X$ is "known", whereas that of $Y$ is not: another interpretation of the problem is that of finding out the degree to which the analytic properties of $Y$ are determined by those of $X$.

The standard approach to the extension problem (Griffiths [14]) is to use the filtration (1.1.1) of $Y$ by the formal neighbourhoods of $X$ in $Y$, so that the problem is broken up into a sequence of extension problems, each of which reduces to the vanishing of a cohomology class on $X$ (the obstructions to extension) or to the vanishing of a cohomology group on $X$ (the obstructions to uniqueness of extension). In practice, the extension of an object to every formal neighbourhood does not necessarily imply extension to $Y$, but this will not be considered here. 
If $A$ is an analytic object on $X$ with an extension $A^{(n)}$ to $X^{(n)}$ for some $n$, then the obstruction to the extension of $A^{(n)}$ to $X^{(n+1)}$ is a cohomology class with coefficients in some coherent analytic sheaf $\mathscr{R}$ on $X$, and it is denoted by $\omega_{n+1}\left(A^{(n)}\right)$. The set of extensions of $A^{(n)}$ to $X^{(n+1)}$ (which is not empty iff $\omega_{n+1}\left(A^{(n)}\right)=0$ ) is denoted by $\Sigma_{n+1}\left(A^{(n)}\right)$. The obstructions to uniqueness will be manifested in the form of a transitive action of another cohomology group on $\Sigma_{n+1}\left(A^{(n)}\right)$ : if $\omega_{n+1}\left(A^{(n)}\right) \in$ $H^{p+1}(X, \mathscr{R})$, then the last group is $H^{p}(X, \mathscr{R})$. When this action is effective, the unique element of $H^{p}(X, \mathscr{R})$ which takes $A_{1}^{(n+1)} \in \Sigma_{n+1}\left(A^{(n)}\right)$ to $A_{2}^{(n+1)} \in$ $\Sigma_{n+1}\left(A^{(n)}\right)$ is denoted by $A_{2}^{(n+1)}-A_{1}^{(n+1)}$.

For the objects of central importance to this article-cohomology classes, bundles, and connections-a more detailed account of the extension problem will now be given.

If $X \hookrightarrow Y$ is defined by $I \subset \mathcal{O}_{Y}$, then one has

$$
0 \rightarrow I^{n+1} / I^{n+2} \rightarrow \mathcal{O}_{X^{(n+1)}} \rightarrow \mathcal{O}_{X^{(n)}} \rightarrow 0,
$$

the fundamental sequence of analytic sheaves on $Y$ supported on $X$. If $E$ is a bundle on $Y$ and $E^{(n)}:=\left.E\right|_{X^{(n)}}$, then the extension problem for an element $\phi^{(n)}$ of $H^{p}\left(X^{(n)}, \mathcal{O}\left(E^{(n)}\right)\right)$ is succinctly described by the exactness of the long cohomology sequence induced by equation $(1.5 .1) \otimes \mathcal{O}\left(E^{(n)}\right): \omega_{n+1}\left(\phi^{(n)}\right)$ is the image of $\phi^{(n)}$ in $H^{p+1}\left(X, \mathcal{O}(E) \otimes I^{n+1} / I^{n+2}\right)$ under the connecting homomorphism; $H^{p}\left(X, \mathcal{O}(E) \otimes I^{n+1} / I^{n+2}\right)$ acts transitively on $\Sigma_{n+1}\left(\phi^{(n)}\right)=$ preimage of $\phi^{(n)}$ in $H^{p}\left(X^{(n+1)}, \mathcal{O}\left(E^{(n+1)}\right)\right)$; etc. Of course, there does exist a global obstruction in $H^{p+1}\left(Y, \mathcal{O}(E) \otimes I^{n+1}\right)$ to the extension of $\phi^{(n)}$ to $Y$, and $H^{p}\left(Y, \mathcal{O}(E) \otimes I^{n+1}\right)$ acts transitively on the set of such extensions, but one is not supposed to knuw anything about cohomology on $Y$.

The case of bundles is essentially the same, except that (1.5.1) is replaced by its nonabelian counterpart

$$
0 \rightarrow M\left(N, I^{n+1} / I^{n+2}\right) \rightarrow \mathrm{GL}\left(N, \mathcal{O}_{X^{(n+1)}}\right) \rightarrow \mathrm{GL}\left(N, \mathcal{O}_{X^{(n)}}\right) \rightarrow 0
$$

(the first map on the left is $m \mapsto 1+m$ ), and the long exact cohomology sequence by its nonabelian analogue (Eastwood [7]). If $B$ is a bundle on $X$ with an extension $B^{(n)}$ to $X^{(n)}$, then

$$
\omega_{n+1}\left(B^{(n)}\right) \in H^{2}\left(X, \mathcal{O}(\text { End } B) \otimes I^{n+1} / I^{n+2}\right),
$$

$H^{1}\left(X, \mathcal{O}(\right.$ End B $\left.) \otimes I^{n+1} / I^{n+2}\right)$ acts transitively on $\Sigma_{n+1}\left(B^{(n)}\right)$, and this action is effective if there exists $B^{(n+1)} \in \Sigma_{n+1}\left(B^{(n)}\right)$ such that

$$
\Gamma\left(X^{(n+1)}, \mathcal{O}\left(\text { End } B^{(n+1)}\right)\right) \rightarrow \Gamma\left(X^{(n)}, \mathcal{O}\left(\text { End } B^{(n)}\right)\right)
$$

is surjective. All these assertions are directly verified using Čech representatives of the various entities involved - this being neither more nor less than the verification of the exactness of the nonabelian cohomology sequence referred to above. Note that even if the action of $H^{1}\left(X, \mathcal{O}(\right.$ End $\left.B) \otimes I^{n+1} / I^{n+2}\right)$ on $\Sigma_{n+1}\left(B^{(n)}\right)$ is not effective, there is nevertheless a canonical choice for the "difference" between two extensions $B_{1}^{(n+1)}, B_{2}^{(n+1)} \in \Sigma_{n+1}\left(B^{(n)}\right)$ : by applying $\operatorname{Hom}\left(B_{2}^{(n+1)}, B_{1}^{(n+1)}\right)$ to (1.5.1) and taking cohomology, the image of

$$
\text { id } \in \Gamma\left(X^{(n)}, \mathcal{O}\left(\text { End } B^{(n)}\right)\right)
$$


in $H^{1}\left(X, \mathcal{O}(\right.$ End $\left.B) \otimes I^{n+1} / I^{n+2}\right)$ is the obstruction to extending id to a homomorphism (which is necessarily an isomorphism) $B_{2}^{(n+1)} \rightarrow B_{1}^{(n+1)}$. This element will always be denoted by $B_{1}^{(n+1)}-B_{2}^{(n+1)}$, irrespective of the effectiveness, or otherwise, of the action.

The case of (relative) connections is slightly more involved: if $E$ is a bundle on $Y$ such that $E^{(n)}:=\left.E\right|_{X^{(n)}}$ has a connection $\nabla^{(n)}$, then since $\nabla^{(n)}$ extends locally to a connection on $E$ and the difference between any two such local extensions is a section of $\mathcal{O}($ End $E) \otimes \mathcal{O}_{Y} \cdot d I^{n+1}$, there is, as for abelian cohomology, a global obstruction to the extension of $\nabla^{(n)}$ to a connection on $E$ lying in

$$
H^{1}\left(Y, \mathcal{O}(\text { End } E) \otimes \mathcal{O}_{Y} \cdot d I^{n+1}\right)
$$

and $H^{0}\left(Y, \mathcal{O}(\right.$ End $\left.E) \otimes \mathcal{O}_{Y} \cdot d I^{n+1}\right)$ acts both transitively and effectively on the set of such extensions. To extend $\nabla^{(n)}$ to a connection on $E^{(n+1)}$, the complete obstruction thus lies in

$$
H^{1}\left(X^{(1)}, S_{X^{(n)}}^{1}\left(\text { End } E^{(1)}\right)\right)
$$

and $H^{0}\left(X^{(1)}, S_{X^{(n)}}^{1}\left(\right.\right.$ End $\left.\left.E^{(1)}\right)\right)$ acts both transitively and effectively on $\Sigma_{n+1}\left(\nabla^{(n)}\right)$ (recall that $S_{X^{(n)}}^{1}:=\operatorname{ker} \Omega_{X^{(n+1)}}^{1} \rightarrow \Omega_{X^{(n)}}^{1}$ and, here, $S_{X^{(n)}}^{1}\left(\right.$ End $\left.E^{(n)}\right):=S_{X^{(n)}}^{1} \otimes$ $\mathcal{O}($ End $E))$. The image of the obstruction in $\left.H^{1}\left(X^{(n+1)}\right), \Omega_{X^{(n+1)}}^{1}\left(\operatorname{End} E^{(n+1)}\right)\right)$ is precisely $D E^{(n+1)}$.

The fact that $S_{X^{(n)}}^{1}$ is a coherent analytic sheaf on $X^{(1)}$, but not on $X$, is not in keeping with the spirit of the extension problem. To circumvent this the extension problem for $\nabla^{(n)}$ must be considered in two stages-namely, the kernels of each of the two epimorphisms $\Omega_{Y}^{1} \otimes \mathcal{O}_{X^{(n)}} \rightarrow \Omega_{X^{(n)}}^{1}$ and $\Omega_{X^{(n+1)}}^{1} \rightarrow \Omega_{Y}^{1} \otimes \mathcal{O}_{X^{(n)}}$ are both coherent analytic sheaves on $X$, and the staggered extension problem consists of first trying to extend $\nabla^{(n)}$ to a map $\tilde{\nabla}^{(n)}: \mathcal{O}\left(E^{(n+1)}\right) \rightarrow \Omega_{Y}^{1}\left(E^{(n)}\right)$ satisfying the appropriate Leibnitz rule and, subsequently, extending any such $\tilde{\nabla}^{(n)}$ to a genuine connection on $E^{(n+1)}$. The two obstructions, lying in $H^{1}$ of the appropriate kernel, are denoted by $\tilde{\omega}_{n+1}\left(\nabla^{(n)}\right)$ and $\omega_{n+1}\left(\tilde{\nabla}^{(n)}\right)$, respectively.

Statements analogous to the above can clearly be made for the extension problem for relative connections. The details need not be repeated.

Until now the spaces $X$ and $Y$ have been arbitrary, but with the assumption that they are both nonsingular, relations between the extension problems for different objects manifest themselves, and, in particular, the extension problem for bundles with connections is considerably simplified: the kernels of the two epimorphisms

$$
\Omega_{Y}^{1} \otimes \mathcal{O}_{X^{(n)}} \rightarrow \Omega_{X^{(n)}}^{1} \text { and } \Omega_{X^{(n+1)}}^{1} \rightarrow \Omega_{Y}^{1} \otimes \mathcal{O}_{X^{(n)}}
$$

are then

$$
I^{n+1} / I^{n+2} \text { and } R_{X^{(n)}}^{2}=\operatorname{coker} I^{n+2} / I^{n+3} \rightarrow \Omega_{Y}^{1} \otimes I^{n+1} / I^{n+2},
$$

respectively, these being the relevant coefficient sheaves for the two stages of the extension problem for connections. 
Proposition H. Suppose $X$ and $Y$ are nonsingular and let $(E, \nabla)$ be a bundle with connection on $X$ with an extension $\left(E^{(n)}, \nabla^{(n)}\right)$ to $X^{(n)}$. Then:

(a) There exists an extension $\left(E^{(n+1)}, \tilde{\nabla}^{(n)}\right)$ of $\left(E^{(n)}, \nabla^{(n)}\right)$, and for any two such extensions there exists a unique isomorphism between them which restricts to the identity on $\left(E^{(n)}, \nabla^{(n)}\right)$.

(b) Under the connecting homomorphisms from the exact sequences

$$
0 \rightarrow R_{X^{(n)}}^{2}(\text { End } E) \rightarrow \Omega_{Y}^{2}\left(\text { End } E^{(n)}\right) \rightarrow \Omega_{X^{(n)}}^{2}\left(\text { End } E^{(n)}\right) \rightarrow 0
$$

and

$0 \rightarrow \mathcal{O}($ End $E) \otimes I^{n+2} / I^{n+3} \rightarrow \Omega_{Y}^{1}($ End $E) \otimes I^{n+1} / I^{n+2} \rightarrow R_{Y^{(n)}}^{2}($ End $E) \rightarrow 0$, we obtain

$$
\begin{aligned}
& H^{0}\left(X^{(n)}, \Omega_{X^{(n)}}^{2}\left(\text { End } E^{(n)}\right)\right) \ni \mathbf{F}\left(\nabla^{(n)}\right) \mapsto \omega_{n+1}\left(\tilde{\nabla}^{(n)}\right) \in H^{1}\left(X, R_{X^{(n)}}^{2}(\text { End } E)\right), \\
& H^{1}\left(X, R_{X^{(n)}}^{2}(\text { End } E)\right) \ni \omega_{n+1}\left(\tilde{\nabla}^{(n)}\right) \\
& \mapsto \omega_{n+2}\left(E^{(n+1)}\right) \in H^{2}\left(X, \mathcal{O}(\text { End }) \otimes I^{n+2} / I^{n+3}\right) .
\end{aligned}
$$

(c) Suppose $\nabla_{1}^{(n+1)}, \nabla_{2}^{(n+1)} \in \Sigma_{n+1}\left(\tilde{\nabla}^{(n)}\right)$. If $E_{1}^{(n+2)}, E_{2}^{(n+2)} \in \Sigma_{n+2}\left(E^{(n+1)}\right)$ are the extensions of $E^{(n+1)}$ determined by $\nabla_{1}^{(n+1)}, \nabla_{2}^{(n+1)}$, respectively, as in (a), then, with the sequences of $(\mathrm{b})$,

$$
\begin{aligned}
H^{0}\left(X, R_{X^{(n)}}^{2}\right. & \text { End } E)) \ni \nabla_{1}^{(n+1)}-\nabla_{2}^{(n+1)} \\
& \left.\mapsto\left(\mathbf{F}\left(\nabla_{1}^{(n+1)}\right)-\mathbf{F}\left(\nabla_{2}^{(n+1)}\right)\right)\right|_{X^{(n)}} \in H^{0}\left(X^{(n)}, \Omega_{Y}^{2}\left(\text { End } E^{(n)}\right)\right)
\end{aligned}
$$

and

$$
\begin{aligned}
H^{0}\left(X, R_{X^{(n)}}^{2}(\text { End } E)\right) & \ni \nabla_{1}^{(n+1)}-\nabla_{2}^{(n+1)} \\
& \mapsto E_{1}^{(n+2)}-E_{2}^{(n+2)} \in H^{1}\left(X, \mathcal{O}(\text { End } E) \otimes I^{n+2} / I^{n+3}\right) .
\end{aligned}
$$

(d) If $\nabla^{(n)}$ is flat, there exists $\left(E^{(n+1)}, \nabla^{(n+1)}\right) \in \Sigma_{n+1}\left(E^{(n)}, \nabla^{(n)}\right)$ such that $\nabla^{(n+1)}$ is flat, and for any two such extensions there is a unique isomorphism between them restricting to the identity on $\left(E^{(n)}, \nabla^{(n)}\right)$.

Proof. (a) One has the fundamental commutative diagram with exact rows

$$
\begin{aligned}
& 0 \rightarrow I^{n+1} / I^{n+2} \rightarrow \Omega_{Y}^{1} \otimes \mathcal{O}_{X^{(n)}} \rightarrow \Omega_{X^{(n)}}^{1} \rightarrow 0 \\
& \uparrow \text { id } \quad \uparrow \tilde{d} \quad \uparrow d \\
& 0 \rightarrow I^{n+1} / I^{n+2} \quad \rightarrow \quad \mathcal{O}_{X^{(n+1)}} \quad \rightarrow \quad \mathcal{O}_{X^{(n)}} \rightarrow 0
\end{aligned}
$$

and its nonabelian analogue

$$
\begin{aligned}
& 0 \rightarrow M\left(\frac{N, I^{n+1}}{I^{n+2}}\right) \quad \rightarrow \quad M\left(N, \Omega_{Y}^{1} \otimes \mathcal{O}_{X^{(n)}}\right) \quad \rightarrow \quad M\left(N, \Omega_{X^{(n)}}^{1}\right) \quad \rightarrow \quad 0 \\
& \uparrow \text { id } \\
& \uparrow \tilde{D} \\
& \uparrow D \\
& 0 \rightarrow M\left(\frac{N, I^{n+1}}{I^{n+2}}\right) \quad \rightarrow \quad \mathrm{GL}\left(N, \mathcal{O}_{X^{(n+1)}}\right) \quad \rightarrow \quad \mathrm{GL}\left(N, \mathcal{O}_{X^{(n)}}\right) \quad \rightarrow \quad 0
\end{aligned}
$$


(where $D g=g^{-1} d g$ as before, with $\tilde{D}$ defined analogously). Tensoring through the top row of (1.5.2) by $\mathcal{O}\left(\mathrm{End}^{(n)}\right)$ and taking cohomology, we see that commutativity of (1.5.3) implies that the image of $D E^{(n)}$ under the connecting homomorphism is precisely $\omega_{n+1}\left(E^{(n)}\right)$, so the existence of $\nabla^{(n)}$ implies $\Sigma_{n+1}\left(E^{(n)}\right) \neq \varnothing$.

If $E^{(n+1)} \in \Sigma_{n+1}\left(E^{(n)}\right)$, the obstruction $\tilde{\omega}_{n+1}\left(\nabla^{(n)}\right)\left(=\tilde{\omega}_{n+1}\left(\nabla^{(n)}, E^{(n+1)}\right)\right)$ to extending $\nabla^{(n)}$ to a map $\tilde{\nabla}^{(n)}: \mathcal{O}\left(E^{(n+1)}\right) \rightarrow \Omega_{Y}^{1}\left(E^{(n)}\right)$ lies in

$$
H^{1}\left(X, \mathcal{O}(\text { End } E) \otimes I^{n+1} / I^{n+2}\right) \text {. }
$$

The action of this group on $\Sigma_{n+1}\left(E^{(n)}\right)$ is translated into

$$
\begin{aligned}
& \tilde{\omega}_{n+1}\left(\nabla^{(n)}, \phi \cdot E^{(n+1)}\right)=\phi+\tilde{\omega}_{n+1}\left(\nabla^{(n)}, E^{(n+1)}\right) \\
& \text { for } \phi \in H^{1}\left(X, \mathcal{O}(\text { End } E) \otimes I^{n+1} / I^{n+2}\right) ;
\end{aligned}
$$

therefore there exists $E^{(n+1)} \in \Sigma_{n+1}\left(E^{(n)}\right)$, which admits an extension $\tilde{\nabla}^{(n)}$ of $\nabla^{(n)}$.

Given two connections $\nabla_{1}^{(n)}, \nabla_{2}^{(n)}$ on $E^{(n)}$ and extensions $\left(E_{i}^{(n+1)}, \tilde{\nabla}_{i}^{(n)}\right)$ of $\left(E^{(n)}, \nabla_{i}^{(n)}\right)$, applying $\operatorname{Hom}\left(E_{2}^{(n+1)}, E_{1}^{(n+1)}\right)$ to (1.5.2), and altering the vertical maps to those induced by $\tilde{\nabla}_{i}$ on $E_{i}^{(n+1)}$, one obtains a new commutative diagram with exact rows. By commutativity,

$$
E_{1}^{(n+1)}-E_{2}^{(n+1)}=\text { image of id }=\text { image of } \nabla_{1}^{(n)}-\nabla_{2}^{(n)}
$$

under the connecting homomorphisms. In particular, if $\nabla_{1}^{(n)}=\nabla^{(n)}=\nabla_{2}^{(n)}$, it follows that $E_{1}^{(n+1)} \simeq E_{2}^{(n+1)}$. Indeed, the commutativity of the diagram implies that there exists a unique isomorphism $\psi: E_{2}^{(n+1)} \rightarrow E_{1}^{(n+1)}$ restricting to the identity on $E^{(n)}$ and satisfying $\tilde{\nabla} \psi=0$ (i.e., $\tilde{\nabla}_{1} \circ \psi=\psi \circ \tilde{\nabla}_{2}$ ).

(b) Under the map induced by the inclusion $R_{X^{(n)}}^{2} \rightarrow \Omega_{X^{(n+1)}}^{1}, \omega_{n+1}\left(\tilde{\nabla}^{(n)}\right)$ is mapped to $D E^{(n+1)}$. By commutativity of the diagram

$$
\begin{aligned}
& 0 \quad \rightarrow \quad I^{n+2} / I^{n+3} \quad \rightarrow \quad \Omega_{Y}^{1} \otimes \mathcal{O}_{X^{(n+1)}} \quad \rightarrow \quad \Omega_{X^{(n+1)}}^{1} \rightarrow 0 \\
& \uparrow \text { id } \uparrow \uparrow \\
& 0 \rightarrow I^{n+2} / I^{n+3} \rightarrow \Omega_{Y}^{1} \otimes I^{n+1} / I^{n+2} \rightarrow R_{X^{(n)}}^{2} \rightarrow 0
\end{aligned}
$$

and the first paragraph of the proof of $(\mathrm{a}), \omega_{n+1}\left(\tilde{\nabla}^{(n)}\right)$ is mapped to $\omega_{n+2}\left(E^{(n+1)}\right)$ by the connecting homomorphism.

The inclusion of $R_{X^{(n)}}^{2}$ in $\Omega_{Y}^{2} \otimes \mathcal{O}_{X^{(n)}}$ leads to another commutative diagram with exact rows analogous to (1.5.2):

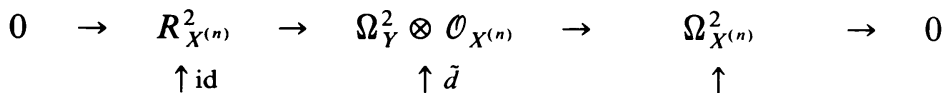

$$
\begin{aligned}
& 0 \quad \rightarrow \quad R_{X^{(n)}}^{2} \rightarrow \Omega_{X^{(n+1)}}^{1} \rightarrow \Omega_{Y}^{1} \otimes \mathcal{O}_{X^{(n)}} \rightarrow 0
\end{aligned}
$$

(indeed, there are obvious analogues of (1.5.4) and (1.5.5) for forms of any degree). Tensoring through (1.5.5) by $\mathcal{O}\left(\right.$ End $\left.E^{(n+1)}\right)$ and changing $\tilde{d}$ to $\tilde{\nabla}^{(n)}$, we see that the new diagram commutes, and it follows that $\omega_{n+1}\left(\tilde{\nabla}^{(n)}\right)$ is the image of $\mathbf{F}\left(\nabla^{(n)}\right)$ under the connecting homomorphism. This completes the proof of (b).

(c) As we observed in the proof of (a),

$$
\nabla_{1}^{(n+1)}-\nabla_{2}^{(n+1)} \in H^{0}\left(X^{(n+1)}, \Omega_{X^{(n+1)}}^{1}\left(\text { End } E^{(n+1)}\right)\right)
$$


is mapped to

$$
E_{1}^{(n+2)}-E_{2}^{(n+2)} \in H^{1}\left(X, \mathcal{O}(\text { End } E) \otimes I^{n+1} / I^{n+2}\right)
$$

under the connecting homomorphism from the top row of (1.5.4) $\otimes \mathcal{O}\left(\right.$ End $\left.E^{(n+1)}\right)$, so the second statement of (c) follows by commutativity of this diagram.

If $\hat{\nabla}$ denotes the connection on End $E^{(n+1)}=\operatorname{Hom}\left(E_{2}^{(n+1)}, E_{1}^{(n+1)}\right)$ induced by $\tilde{\nabla}_{1}^{(n+1)}$ and $\tilde{\nabla}_{2}^{(n+1)}$, then, after tensoring through (1.5.5) by $\mathcal{O}\left(\right.$ End $\left.E^{(n+1)}\right)$ and altering the vertical maps to those induced by $\hat{\nabla}$, the new diagram commutes. The first statement of (c) then follows from the observation that

$$
\begin{aligned}
\hat{\nabla}^{2}(\mathrm{id}) & =\hat{\nabla}\left(\nabla_{1}^{(n+1)}-\nabla_{2}^{(n+1)}\right)=\mathbf{F}\left(\nabla_{1}^{(n+1)}\right)-\mathbf{F}\left(\nabla_{2}^{(n+1)}\right) \\
& \in H^{0}\left(X^{(n+1)}, \Omega_{X^{(n+1)}}^{2}\left(\text { End } E^{(n+1)}\right)\right) .
\end{aligned}
$$

(d) If $\nabla^{(n)}$ is flat, then by (b), $\Sigma_{n+1}\left(\nabla^{(n)}\right) \neq \varnothing$. By commutativity of $(1.5 .5) \otimes$ $\mathcal{O}\left(E^{(n+1)}\right)$, there exists $\nabla^{(n+1)} \in \Sigma_{n+1}\left(\nabla^{(n)}\right)$ such that the composition

$$
\mathcal{O}\left(E^{(n+1)}\right) \rightarrow \Omega_{X^{(n+1)}}^{1}\left(E^{(n+1)}\right) \rightarrow \Omega_{Y}^{2}\left(E^{(n)}\right)
$$

is zero, i.e. $\left.\mathbf{F}\left(\nabla^{(n+1)}\right)\right|_{X^{(n)}}=0$. Hence, the composition

$$
\Omega_{X^{(n+1)}}^{1}\left(E^{(n+1)}\right) \rightarrow \Omega_{X^{(n+1)}}^{2}\left(E^{(n+1)}\right) \rightarrow \Omega_{Y}^{3}\left(E^{(n)}\right)
$$

is also zero, and by commutativity of the analogue of $(1.5 .5) \otimes \mathcal{O}\left(E^{(n+1)}\right)$ for forms of the next higher degree, it follows immediately that $\mathbf{F}\left(\nabla^{(n+1)}\right)$ vanishes. Thus $E^{(n+1)}$ admits a flat extension of $\nabla^{(n)}$, and the uniqueness statement is proved in the obvious way.

Thus, up to isomorphism, $\left(E^{(n)}, \nabla^{(n)}\right)$ extends uniquely to all orders. This is, of course, a reflection of the empty statement that an element of $H^{1}(X, \operatorname{GL}(N, \mathbf{C}))$ extends to $X^{(n)}$ for all $n$.

With a purely formal change of notation and the insertion of the word "relative" in front of "connection", Proposition $\mathrm{H}$ can be repeated for the case of relative connections in the circumstances of Proposition B (i.e., $F: Y \rightarrow V, f: X \rightarrow V$ surjective of maximal rank with $\left.F\right|_{X}=f$ ). Indeed, this relative form of the proposition is the one used most, if not exclusively, in the applications in Part II.

The formalism of the extension problem can be used to give new proofs of (or at least different interpretations of the proofs of) Propositions $F$ and $G$ on inverting pull-back functors. The case of Proposition $F$ is of particular relevance in this context: with the notation and hypotheses of that proposition, it was shown that the functor $f^{(n, m)^{*}}$ defines an equivalence of the category of bundles $B^{(m)}$ on $V^{(m)}$ with the category of bundles $E^{(n, m)}$ with flat relative connection $\nabla_{f^{(n, m)}}$ on $X^{(n, m)}$. Thus the extension problem for a bundle $B$ on $V$ must be equivalent to the extension problem for $\left(f^{(n)^{*}} B, d_{f^{(n)}}\right)$ to $X^{(n, m)}$ for any $n \geqslant m$.

To illustrate this, given $\left(E^{(n)}, \nabla_{f^{(n)}}\right)$ on $X^{(n)}$ with $\nabla_{f^{(n)}}$ flat, construct $B$ on $V$ with $\left.\left(f^{*} B, d_{f}\right) \simeq\left(E^{(n)}, \nabla_{f^{(n)}}\right)\right|_{X}$, as in the proof of Proposition F. Then the relative form of part (d) of Proposition $\mathrm{H}$ and induction give a unique isomorphism $\left(f^{(n)^{*}} B, d_{f^{(n)}}\right)$ $\simeq\left(E^{(n)}, \nabla_{f^{(n)}}\right)$ restricting to the initial isomorphism on $X$. 
As another example, suppose that $B$ is a bundle on $V$ with an extension $B^{(m)}$ to $V^{(m)}$, and set $E^{(n, m)}:=f^{(n, m)^{*}} B^{(m)}$. Then, in chasing $\omega_{m+1}\left(B^{(m)}\right)$ through the cohomology of the resolution

$$
0 \rightarrow f^{-1} \mathcal{O}(\text { End } B) \otimes J_{V}^{m+1} / J_{V}^{m+2} \rightarrow \Omega_{f^{(n-m-1)}}\left(\text { End } E^{(n-m-1)}\right) \otimes J^{m+1} / J^{m+2},
$$

one encounters three consecutive "obstructions" to the vanishing of $\omega_{m+1}\left(B^{(m)}\right)$. In order, these are elements of

$$
\begin{gathered}
H^{2}\left(X, \mathcal{O}\left(\text { End } E^{(n-m-1)}\right) \otimes J^{m+1} / J^{m+2}\right) ; \\
H^{1}\left(X, \Omega_{f^{(n-m-1)}}^{1}\left(\text { End } E^{(n-m-1)}\right) \otimes J^{m+1} / J^{m+2}\right) \\
{\left[\bmod d_{f^{(n-m-1)}} H^{1}\left(X, \mathcal{O}\left(\text { End } E^{(n-m-1)}\right) \otimes J^{m+1} / J^{m+2}\right)\right] ;} \\
H^{0}\left(X, \Omega_{f^{(n-m-1)}}^{2}\left(\text { End } E^{(n-m-1)}\right) \otimes J^{m+1} / J^{m+2}\right) \\
{\left[\bmod d_{f^{(n-m-1)}} H^{0}\left(X, \Omega_{f^{(n-m-1)}}^{1}\left(\text { End } E^{(n-m-1)}\right) \otimes J^{m+1} / J^{m+2}\right)\right] .}
\end{gathered}
$$

The first of these is $\omega_{m+1}\left(E^{(n, m)}\right)$, the second is the obstruction to extending $d_{f^{(n, m)}}$ on $E^{(n, m)}$ to a relative connection on $E^{(n, m+1)} \in \Sigma_{m+1}\left(E^{(n, m)}\right)$ [mod choice of $\left.E^{(n, m+1)}\right]$, and the third is (essentially) the curvature of any such $\nabla_{f^{(n, m+1)}} \in$ $\Sigma_{m+1}\left(d_{f^{(n, m)}}\right)$ [mod choice of $\nabla_{f^{(n, m+1)}}$ ]. These last two assertions are easily verified by chasing the appropriate parts of $(1.3 .12) \otimes \mathcal{O}\left(E^{(n, m+1)}\right)$.

As a final comment before moving on, it is worth noting that differential forms on analytic spaces can be used for extension problems other than just those considered here. For example, if $X$ and $Y$ are as above, and $g: X \rightarrow V^{\prime}$ is an arbitrary holomorphic mapping to a manifold $V^{\prime}$ with an extension $g^{(n)}: X^{(n)} \rightarrow V^{\prime}$ for some $n$, then, after applying $\operatorname{Hom}\left(g^{(n)^{*}} \Omega_{V^{\prime}}^{1},-\right)$ to the top row of (1.5.2) and taking cohomology,

$$
\omega_{n+1}\left(g^{(n)}\right) \in H^{1}\left(X, \operatorname{Hom}\left(g^{*} \Omega_{V^{\prime}}^{1}, I^{n+1} / I^{n+2}\right)\right)
$$

is the image of $d g^{(n)}$ under the connecting homomorphism, $\Sigma_{n+1}\left(g^{(n)}\right)$ is in 1-1 correspondence with the preimage of $d g^{(n)}$ in $H^{0}\left(X, \operatorname{Hom}\left(g^{(n)^{*}} \Omega_{V^{\prime}}^{1}, \Omega_{Y}^{1} \otimes \mathcal{O}_{X^{(n)}}\right)\right)$, and the transitive and effective action of $H^{0}\left(X, \operatorname{Hom}\left(g^{*} \Omega_{V^{\prime}}^{1}, I^{n+1} / I^{n+2}\right)\right)$ on $\Sigma_{n+1}\left(g^{(n)}\right)$ is simply reinterpreted as the exactness of the first part of the long cohomology sequence.

\section{PART II : NON-SELF-DUAL YANG-MILlS FIELDS}

The various pieces of machinery required for generalizing the method of double fibrations to analytic spaces have been established in Part I. Part II begins with a discussion of the particular class of spaces used in the application of the generalized method to Yang-Mills theory.

2.1. Flag manifolds. Let $V$ be an $n$-dimensional complex vector space. For any sequence of integers $0<d_{1}<\cdots<d_{s}<n$, denote by $\mathbb{F}_{d_{1}, \ldots, d_{s}}(V)$ the set of $s$-tuples $\left(L_{1}, \ldots, L_{d_{s}}\right)$, where $L_{d_{i}}$ is a $d_{i}$-dimensional linear subspace of $V$ with $L_{d_{i}} \subset L_{d_{i+1}}$. The natural action of $\mathrm{GL}(V)$ on this set is transitive and endows it with the structure of a complex manifold-i.e., the homogeneous space $\mathrm{GL}(V) /$ (isotropy group of a point). Natural coordinate systems for the manifold are determined by a 
choice of basis for $V$. The generic term for such objects is flag manifolds; for $s=1$, $\mathbb{F}_{d}(V)$ is the Grassmannian of $d$-planes in $V$, which, for $d=1$, is the familiar $\mathbb{P}(V)$ - projectivized $V$. A choice of hermitian inner product on $V$ gives rise to a description of $\mathbb{F}_{d_{1} \ldots d_{s}}(V)$ as a quotient of unitary groups by closed subgroups, so $\mathbb{F}_{d_{1} \ldots, d_{s}}(V)$ is a compact complex manifold (of dimension $d_{1}\left(d_{2}-d_{1}\right)+$ $\left.d_{2}\left(d_{3}-d_{2}\right)+\cdots+d_{s}\left(n-d_{s}\right)\right)$.

For any ordered subset $\left\{e_{1}, \ldots, e_{t}\right\} \subset\left\{d_{1}, \ldots, d_{s}\right\}$, there is a "projection" $\mathbb{F}_{d_{1}, \ldots d_{s}}(V) \rightarrow \mathbb{F}_{e_{1} \ldots, e_{t}}(V)$ given by $\left(L_{d_{1}}, \ldots, L_{d_{s}}\right) \rightarrow\left(L_{e_{1}}, \ldots, L_{e_{t}}\right)$ which is a surjective holomorphic mapping of maximal rank. The fibre of such a mapping is a finite product of flag manifolds, and the mapping expresses $\mathbb{F}_{d_{1} \ldots, d_{s}}(V)$ as a locally trivial holomorphic fibration over $\mathbb{F}_{e_{1} \ldots, e_{t}}(V)$.

There are embeddings $\mathbb{F}_{d_{1} \ldots d_{s}}(V) \hookrightarrow \mathbb{F}_{d_{1}}(V) \times \cdots \times \mathbb{F}_{d_{s}}(V), \mathbb{F}_{d}(V) \hookrightarrow \mathbb{F}_{1}\left(\bigwedge^{d} V\right)$ (given by $\left.L_{d} \mapsto \wedge^{d} L_{d}\right)$ and $\mathbb{F}_{1}(V) \times \mathbb{F}_{1}\left(V^{\prime}\right) \hookrightarrow \mathbb{F}_{1}\left(V \otimes V^{\prime}\right.$ ) (given by $\left(L_{1}, L_{1}^{\prime}\right) \mapsto L_{1}$ $\left.\otimes L_{1}^{\prime}\right)$, so all flag manifolds are algebraic.

For each $i$ there is a rank $d_{i}$ homogeneous vector bundle $E_{d_{i}}$ on $\mathbb{F}_{d_{1} \ldots, d_{s}}(V)$, the fibre of which at $\left(L_{d_{1}}, \ldots, L_{d_{s}}\right)$ is the vector space $L_{d_{i}} . E_{d_{i}}$ is a subbundle of $E_{d_{j}}$ for $i<j$, and all are subbundles of the trivial bundle $V$ on $\mathbb{F}_{d_{1} \ldots, d_{s}}(V)$. Using the standard operations of linear algebra, we may construct many more such homogeneous vector bundles: direct sums, duals tensor products, quotients, etc. For example, the tangent bundle of the Grassmannian $\mathbb{F}_{d}(V)$ is canonically isomorphic to $\operatorname{Hom}\left(E_{d}, V / E_{d}\right)=E_{d}^{*} \otimes V / E_{d}$. In [3] Bott gives a prescription for explicitly calculating the global cohomology $H^{*}\left(\mathbb{F}_{d_{1} \ldots d_{s}}(V), \mathcal{O}(-)\right)$ of all these bundles, the most well-known application of which is Bott's Rule: on $\mathbb{P}(V), \mathcal{O}\left(\otimes{ }^{k} E_{1}\right)$ is conventionally denoted by $\mathcal{O}(-k)$ and its dual by $\mathcal{O}(k)$, and the result is

$$
H^{p}(\mathbb{P}(V), \mathcal{O}(k)) \simeq \begin{cases}\odot^{k} V^{*}, & p=0, k \geqslant 0, \\ \operatorname{det} V \otimes \odot^{-k-n} V, & p=n-1, k \leqslant-n, \\ 0 & \text { otherwise. }\end{cases}
$$

(Here $\odot$ denotes symmetric tensor product and $\operatorname{det} V:=\wedge^{n} V$.)

Note that there is a canonical isomorphism $\mathbb{F}_{d_{1} \ldots, d_{s}}(V) \stackrel{\sim}{\rightarrow} \mathbb{F}_{n-d_{s}, \ldots, n-d_{1}}\left(V^{*}\right)$ given by $\left(L_{d_{1}}, \ldots, L_{d_{s}}\right) \mapsto\left(L_{d_{s}}^{\perp}, \ldots, L_{d_{1}}^{\perp}\right)$, where $L_{d_{i}}^{\perp} \simeq\left(V / L_{d_{i}}\right)^{*}$ is the annihilator of $L_{d_{i}}$.

The case of $\operatorname{dim} V=4$ is the case of particular concern, and here there are a number of conventional symbols used for the various objects defined in generality above. First and foremost, the symbol $T$ (for twistor space) is used in place of $V$. For the various flag manifolds derived from $T$, the Grassmannian $\mathbb{F}_{2}(T)$ is denoted by $M$ (for complexified, compactified Minkowski space), $\mathbb{F}_{1}(T)$ by $P$ (projective twistor space), $\mathbb{F}_{3}(T)\left(\simeq \mathbb{F}_{1}\left(T^{*}\right)\right)$ by $P^{*}, \mathbb{F}_{1,3}(T)$ by $A$ (ambitwistor space), and finally $\mathbb{F}_{1,2,3}(T)$ by $F$. If $a: F \rightarrow A$ and $b: F \rightarrow M$ are the "projections", then the following diagram indicates the general set-up which is the framework for Part II.

$$
\begin{aligned}
& F \stackrel{I}{\hookrightarrow} M \times A \quad \stackrel{J}{\hookrightarrow} \quad M \times P \times P^{*} \\
& b \swarrow \quad \searrow a \quad \swarrow \text { proj } \quad \swarrow \text { proj } \\
& M \quad A \quad \stackrel{J_{A}}{\hookrightarrow} \quad P \times P^{*}
\end{aligned}
$$


The notation for the basic homogeneous vector bundles is as follows: $\mathcal{O}(-1,0):=\mathcal{O}\left(E_{1}\right)$ and $\mathcal{O}(0,-1):=\mathcal{O}\left(\left(T / E_{3}\right)^{*}\right)$, the integers in brackets being essentially the first Chern classes of these line bundles. Tensor products are denoted by adding the pairs of integers together; duals, by changing the signs. For example,

$$
\mathcal{O}(p, q):=\mathcal{O}\left(\otimes^{p} E_{1}^{*}\right) \otimes \mathcal{O}\left(\otimes^{q} T / E_{3}\right) .
$$

The symbol $S^{\prime}$ (for primed spin bundle) denotes $E_{2}$, and $S$ denotes $\left(T / E_{2}\right)^{*}$ (the pull-back of $E_{2}$ on $\mathbb{F}_{2}\left(T^{*}\right)$ under the isomorphism $\mathbb{F}_{2}(T) \stackrel{\sim}{\rightarrow} \mathbb{F}_{2}\left(T^{*}\right)$ is $\left.S\right)$. Finally, $\mathcal{O}\left(\operatorname{det} S^{\prime}\right)=: \mathcal{O}[-1]^{\prime}$ and $\mathcal{O}(\operatorname{det} S)=: \mathcal{O}[-1]$, with $\mathcal{O}[p][q]^{\prime}$ defined in the obvious way. Note that $\operatorname{det} S=\operatorname{det} S^{\prime} \otimes \operatorname{det} T^{*}$, so $\mathcal{O}[-1]$ is isomorphic to $\mathcal{O}[-1]^{\prime}$, but not canonically, and the distinction will therefore be retained.

The symbols used to denote the various homogeneous vector bundles have been given without reference to a particular space. Where this is necessary, it is indicated by a subscript; for example, $\mathcal{O}_{F}(p, q)=a^{*} \mathcal{O}_{A}(p, q)$. Similarly, it is convenient to denote the pull-backs to $M \times P \times P^{*}$ of any of the other standard globally-defined sheaves by the same symbol; thus $\Omega_{M} \otimes \mathcal{O}_{F}$ means $b^{*} \Omega_{M}$, for example.

The notation for the various sheaves of ideals defining the inclusions of (2.1.1) will be the same as in $\S 1.3: I \subset \mathcal{O}_{M \times A}$ defines $F \hookrightarrow M \times A, J \subset \mathcal{O}_{M \times P \times P^{*}}$ defines $M \times A \hookrightarrow M \times P \times P^{*}, K \subset \mathcal{O}_{M \times P \times P^{*}}$ defines $F \hookrightarrow M \times P \times P^{*}$, and $J_{A} \hookrightarrow \mathcal{O}_{P \times P^{*}}$ defines $A \hookrightarrow P \times P^{*}$. The sheaves $J / J^{2}, K / K^{2}$, and $J_{A} / J_{A}^{2}$ are canonically identified with sheaves of sections of certain homogeneous vector bundles. For example, if $c$ : $A \rightarrow P^{*}$ is the "projection", the fibre of $c$ over $L_{3} \in P^{*}$ is $\mathbb{P}\left(L_{3}\right)$, and, therefore, $\Omega_{c}^{1}=\mathcal{O}_{A}\left(\left(E_{3} / E_{1}\right)^{*} \otimes E_{1}\right)$. The exact sequence $0 \rightarrow J_{A} / J_{A}^{2} \rightarrow \Omega_{p}^{1} \otimes \mathcal{O}_{F} \rightarrow \Omega_{c}^{1} \rightarrow 0$ then identifies $J_{A} / J_{A}^{2}$ with $\mathcal{O}_{A}\left(\left(T / E_{3}\right)^{*} \otimes E_{1}\right)=\mathcal{O}_{A}(-1,-1)$. Similarly,

$$
J / J^{2}=\mathcal{O}_{F}(-1,-1) \text { and } K / K^{2}=\mathcal{O}_{F}(S)(-1,0) \oplus \mathcal{O}_{F}\left(S^{\prime}\right)(0,-1) .
$$

The identification of the sheaves $J / J^{2}$ and $K / K^{2}$, used in conjunction with the standard manipulations of tensor algebra, and Bott's Rule enable one to compute all of the direct images in the following sections for any homogeneous vector bundle $E$ on $F ; b_{*}^{q} E$ is a homogeneous vector bundle on $M$ with fibre $H^{q}\left(b^{-1}(x), \mathcal{O}\left(\left.E\right|_{b^{-1}(x)}\right)\right)$ at $x \in M$. Because there is a large number of such calculations to be made, the details will usually be suppressed, but the reader is reminded of the Projection Formula

$$
b_{*}^{q}\left(b^{*} B \otimes E\right)=B \otimes b_{*}^{q} E
$$

for any bundle $B$ on $M$, which is indispensable for these calculations.

The canonical isomorphism $\Omega_{M}^{1} \simeq \mathcal{O}\left(S \otimes S^{\prime}\right)$ gives rise to identifications

$$
\begin{gathered}
\Omega_{M}^{2}=\mathcal{O}\left(\odot^{2} S\right)[-1]^{\prime} \oplus \mathcal{O}\left(\odot^{2} S^{\prime}\right)[-1], \\
\Omega_{M}^{3} \simeq \mathcal{O}\left(S \otimes S^{\prime}\right)[-1][-1]^{\prime}, \quad \text { and } \Omega_{M}^{4} \simeq \mathcal{O}[-2][-2]^{\prime} .
\end{gathered}
$$

Denote by $\Omega_{+}^{2}$ and $\Omega_{-}^{2}$, respectively, the two direct summands in the splitting of $\Omega_{M}^{2}$, and define an endomorphism $*$ of $\Omega_{M}^{2}$ so that $* 2=+1$ and $\Omega_{ \pm}^{2}$ is the \pm eigenspace of $*$. The reasoning for this rather suggestive notation is as follows: the identification

$$
\odot^{2} \Omega_{M}^{1}=\mathcal{O}\left(\odot^{2} S\right) \otimes \mathcal{O}\left(\odot^{2} S^{\prime}\right) \oplus \mathcal{O}[-1][-1]^{\prime}
$$


means that $\odot^{2} \Omega_{M}^{1}[1][1]^{\prime}$ has a canonical global section, thus giving a conformal metric on $M$. A Hodge *-operator on an even-dimensional manifold is always conformally invariant on forms of the middle degree, and the splitting $\Omega_{M}^{2} \simeq \Omega_{+}^{2} \oplus \Omega_{-}^{2}$ is just that induced by the conformal metric on $M$. For more details see Le Brun [20].

Finally, the double fibration appearing on the left-hand side of (2.1.1) gives a correspondence between the points of $M$ and certain closed submanifolds of $A$ and also a correspondence between the points of $A$ and certain closed submanifolds of $M$. Specifically, if $x \in M$ is given by $L_{2} \subset T$, the restriction of $a$ to $b^{-1}(x) \simeq \mathbb{P}\left(L_{2}\right)$ $\times \mathbb{P}\left(T / L_{2}\right)$ defines an embedding of this manifold in $A$, and the submanifold $a\left(b^{-1}(x)\right)$ is denoted by $Q_{x}$. Similarly, if $y \in A$ is given by $\left(L_{1}, L_{3}\right) \subset T \times T$, the restriction of $b$ to $a^{-1}(y) \simeq \mathbb{P}\left(L_{3} / L_{1}\right)$ embeds this manifold in $M$. The submanifold $b\left(a^{-1}(y)\right)$, denoted by $N_{y}$, is called a null line (the reason being that it is a null geodesic of the conformal metric on $M$ referred to above).

2.2. The Witten-Isenberg-Yasskin-Green correspondence. For an open subset $U$ of $M$, the category of Yang-Mills bundles $\left(E_{M}, \nabla\right)$ on $U$ is just the category of holomorphic vector bundles with holomorphic connection on $U$. The field $\mathbf{F}$ of $\left(E_{M}, \nabla\right)$ is the curvature $\mathbf{F}(\nabla) \in \Gamma\left(U, \Omega_{M}^{2}\left(\right.\right.$ End $\left.\left.E_{M}\right)\right)$ of the connection; it is said to satisfy the (complex) Yang-Mills equations if $\nabla * \mathbf{F}=0$ ( $\nabla$ denotes the induced connection on End $E_{M}$ as usual). The field is called self-dual (resp., anti-self-dual) if $* \mathbf{F}=\mathbf{F}$ (resp., $* \mathbf{F}=-\mathbf{F}$ ), in which case the Yang-Mills equations are automatically satisfied by virtue of the Bianchi identity $\nabla \mathbf{F}=0$. If $\mathbf{F}_{ \pm}$denotes the \pm component of $\mathbf{F}$ in terms of the splitting $\Omega_{M}^{2}\left(\right.$ End $\left.E_{M}\right)=\Omega_{+}^{2}\left(\right.$ End $\left.E_{M}\right) \oplus \Omega_{-}^{2}\left(\right.$ End $\left.E_{M}\right)$, then the equations can also be written in the form $\nabla \mathbf{F}_{ \pm}=0$, the field being self-dual if $\mathbf{F}_{-}=0$.

For an arbitrary Yang-Mills bundle $\left(E_{M}, \nabla\right)$ on $U$, the section

$$
\mathbf{J}:=\nabla * \mathbf{F} \in \Gamma\left(U, \Omega_{M}^{3}\left(\text { End } E_{M}\right)\right)
$$

is nonzero, in general, and is called the current. Analogous to the Bianchi identity, there is an identity $\nabla \mathbf{J}=0$.

Now let $U^{\prime}:=b^{-1}(U)$ and $U^{\prime \prime}:=a\left(U^{\prime}\right)$, so the double fibration appearing on the left-hand side of (2.1.1) restricts to a double fibration

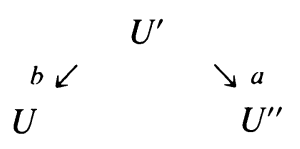

Here, $a$ and $b$ denote the restrictions to $U^{\prime}$ of the appropriate maps; we use this convention when the underlying subset $U \subset M$ is understood.

If $\left(E_{M}, \nabla\right)$ is a Yang-Mills bundle on $U$, let $E_{F}:=b^{*} E_{M}$, and let $\nabla_{a}: \mathcal{O}\left(E_{F}\right) \rightarrow$ $\Omega_{a}^{1}\left(E_{F}\right)$ be the induced relative connection. Since the fibres of $a$ are one dimensional, $\nabla_{a}$ is necessarily flat. Thus, if $a\left(=\left.a\right|_{U^{\prime}}\right)$ has fibres which are all connected and simply connected, Proposition $\mathrm{F}$ applies to give a (unique) bundle $E_{A}$ on $U^{\prime \prime}$ such that $\left(a^{*} E_{A}, d_{a}\right)=\left(E_{F}, \nabla_{a}\right)$. The fibre of $E_{A}$ at $y \in U^{\prime \prime}$ is canonically isomorphic to the vector space of covariantly constant sections of $\left.E_{M}\right|_{N_{y} \cap U}$, which in turn is 
canonically isomorphic to the fibre of $E_{M}$ at $x$ for any $x \in N_{y} \cap U$. Thus, if $x \in U$ is fixed, $E_{A, y}$ is identified with $E_{M, x}$ for any $y \in Q_{x}$, and, therefore, $\left.E_{A}\right|_{Q_{x}}$ is trivial.

To invert this construction, suppose that $E_{A}$ is a bundle on $U^{\prime \prime}$ with the property that its restriction to $Q_{x} \hookrightarrow U^{\prime \prime}$ is trivial for every $x \in U$. Setting $E_{F}:=a^{*} E_{A}$, we see that this property means that

$$
\operatorname{dim} H^{0}\left(b^{-1}(x), \mathcal{O}\left(\left.E_{F}\right|_{b^{-1}(x)}\right)\right)=\operatorname{rank} E_{A}
$$

is independent of $x \in U$, and, therefore, $b_{*} \mathcal{O}\left(E_{F}\right)$ is a locally free analytic sheaf on $U$, thereby defining a bundle $E_{M}$ with $b^{*} E_{M}=E_{F}$.

Since $\Omega_{a}^{1}=\mathcal{O}_{F}(1,1)[-1],[-1]^{\prime}$, Bott's Rule gives

$$
b_{*} \Omega_{a}^{1}=\mathcal{O}_{M}\left(S^{*} \otimes S^{*}\right)[-1][-1]^{\prime}=\Omega_{M}^{1},
$$

and a connection $\nabla$ on $E_{M}$ is induced by $d_{a}$ on $E_{F}$ via

$$
\begin{array}{cc}
b_{*} \mathcal{O}\left(E_{F}\right) & \\
\| & b_{*} \Omega_{a}^{1}\left(E_{F}\right) \\
\mathcal{O}\left(E_{M}\right) \cdots \nabla & \Omega_{M}^{1}\left(E_{M}\right),
\end{array}
$$

where the isomorphism on the right follows from the Projection Formula. By construction the relative connection on $E_{F}$ induced by $\nabla$ on $E_{M}$ agrees with $d_{a}$, so it follows that these two procedures are inverses of each other.

A bundle on $U^{\prime \prime}$ with the property that its restriction to $Q_{x}$ is trivial for every $x \in U$ will henceforth be called $Q$-trivial. The assignment $E_{A} \mapsto\left(E_{M}, \nabla\right)$ defines a functor from the category of $Q$-trivial bundles on $U^{\prime \prime}$ to the category of Yang-Mills bundles on $U$. This functor is compatible with the operations of tensor algebra and restrictions to open subsets of $U$, it is injective when the intersection of $U$ with every null line in $M$ is connected, and it is bijective when every such intersection is both connected and simply connected.

The interpretation of the Yang-Mills field and current of $\left(E_{M}, \nabla\right)$ in terms of $E_{A}$ are the subjects of investigation in the next two sections. To close this section the relationship between the analytic properties of $E_{A}$ and $\left(E_{M}, \nabla\right)$ will be considered in the context of the generalized Penrose transform for cohomology resulting from the construction given so far.

If $B$ is any homogeneous vector bundle on $A$ and $q \geqslant 0$, the relative connection $d_{a}$ on $a^{*} B$ induces a map $b_{*}^{q} \mathcal{O}\left(a^{*} B\right) \rightarrow b_{*}^{q} \Omega_{a}^{1}\left(a^{*} B\right)$ which can be interpreted as a first order linear differential operator acting between homogeneous vector bundles on $M$. For example, if $\mathcal{O}(B)=\mathcal{O}_{A}(-3,-3)$, Bott's Rule gives $b_{*}^{2} \mathcal{O}_{F}(-3,-3) \simeq \Omega_{M}^{3}$ and $b_{*}^{2} \Omega_{a}^{1}(-3,-3) \simeq \Omega_{M}^{4}$ (with all other direct images vanishing), the induced map being $d: \quad \Omega_{M}^{3} \rightarrow \Omega_{M}^{4}$. For $\mathcal{O}(B)=\mathcal{O}_{A}(-2,-2), \quad b_{*}^{2} \mathcal{O}_{F}(-2,-2)=\mathcal{O}_{M}[-1][-1]^{\prime}$ and $b_{*}^{2} \Omega_{a}^{1}(-2,-2)=0$, so the induced operator is zero in this case. As a third example, for $\mathcal{O}(B)=\Omega_{p^{*}}^{3}\left(:=c^{*} \Omega_{p^{*}}^{3}\right.$, where $c: A \rightarrow P^{*}$ is the projection), $b_{*}^{1} \Omega_{p^{*}}^{3} \otimes \mathcal{O} / I=\Omega_{+}^{2}$, $b_{*}^{1} \Omega_{a}^{1} \otimes \Omega_{p^{*}}^{3}=\Omega_{M}^{3}$, and the induced operator is now $d: \Omega_{+}^{2} \rightarrow \Omega_{M}^{3}$. Any number of other examples can easily be given (see e.g. Eastwood [6]), although the notation, involving large tensor products of $S$ and $S^{\prime}$, becomes unwieldy at times. 
If $U \subset M$ is open, $E_{A}$ is a $Q$-trivial bundle on $U^{\prime \prime}$, and $\left(E_{M}, \nabla\right)$ is the corresponding Yang-Mills bundles on $U$, then the above can be repeated with $B$ replaced by $E_{A} \otimes B$. By the Projection Formula,

$$
b_{*}^{q} \mathcal{O}\left(a^{*} E_{A} \otimes B\right)=\mathcal{O}\left(E_{M}\right) \otimes b_{*}^{q}\left(a^{*} B\right)
$$

and

$$
b_{*}^{q} \Omega_{a}^{1}\left(a^{*} E_{A} \otimes B\right)=\mathcal{O}\left(E_{M}\right) \otimes b_{*}^{q} \Omega_{a}^{1}\left(a^{*} B\right),
$$

where the induced operator is induced by $\nabla$ on $E_{M}$ together with the operator $b_{*}^{q} \mathcal{O}\left(a^{*} B\right) \rightarrow b_{*}^{q} \Omega_{a}^{1}\left(a^{*} B\right)$. For example,

$$
\Omega_{M}^{3}\left(E_{M}\right)=b_{*}^{2} \mathcal{O}\left(E_{F}\right)(-3,-3) \rightarrow b_{*}^{2} \Omega_{a}^{1}\left(E_{F}\right)(-3,-3)=\Omega_{M}^{4}\left(E_{M}\right)
$$

is just $\nabla: \Omega_{M}^{3}\left(E_{M}\right) \rightarrow \Omega_{M}^{4}\left(E_{M}\right)$.

A version of the Penrose transform [10] can now be put together: given $B$ and $E_{A}$ as above, the first part of the transform consists of the homomorphism

$$
H^{p}\left(U^{\prime \prime}, \mathcal{O}\left(E_{A} \otimes B\right)\right) \rightarrow H^{p}\left(U^{\prime \prime}, a^{-1} \mathcal{O}\left(E_{A} \otimes B\right)\right) ;
$$

the second consists of expressing the latter group in terms of analytic cohomology on $U^{\prime}$ by using the exact sequence

$$
0 \rightarrow a^{-1} \mathcal{O}\left(E_{A} \otimes B\right) \rightarrow \mathcal{O}\left(E_{F} \otimes a^{*} B\right) \rightarrow \Omega_{a}^{1}\left(E_{F} \otimes a^{*} B\right) \rightarrow 0 ;
$$

the third consists of "evaluating" the groups $H^{*}\left(U^{\prime}, \Omega_{a}^{*}\left(E_{F} \otimes a^{*} B\right)\right)$ in terms of analytic cohomology on $U$ using the Leray spectral sequence (usually highly degenerate) and expressing the maps between the groups on $U$ in terms of the previously identified differential operators. For example, with $\mathcal{O}(B)=\mathcal{O}_{A}(-3,-3)$, the complete transform is

$$
\begin{array}{ccc}
H^{2}\left(U^{\prime \prime}, \mathcal{O}\left(E_{A}\right)(-3,-3)\right) & \rightarrow H^{2}\left(U^{\prime}, a^{-1} \mathcal{O}\left(E_{A}\right)(-3,-3)\right) & \\
=\operatorname{ker} H^{2}\left(U^{\prime}, \mathcal{O}\left(E_{F}\right)(-3,-3)\right) & \rightarrow & H^{2}\left(U^{\prime}, \Omega_{a}^{1}\left(E_{F}\right)(-3,-3)\right) \\
\| & & \| \\
H^{0}\left(U, \Omega_{M}^{3}\left(E_{M}\right)\right) & \stackrel{\nabla}{\rightarrow} & H^{0}\left(U, \Omega_{M}^{4}\left(E_{M}\right)\right) .
\end{array}
$$

By Proposition $\mathrm{E}$ the topmost arrow is injective if the intersection of $U$ with every null line in $M$ is connected and simply connected; moreover, it is an isomorphism if these intersections are noncompact. The transform for $\mathcal{O}(B)=\Omega_{p^{*}}^{3}$,

$$
H^{1}\left(U^{\prime \prime}, \Omega_{p^{*}}^{3}\left(E_{A}\right)\right) \rightarrow \operatorname{ker} \nabla: H^{0}\left(U, \Omega_{+}^{2}\left(E_{M}\right)\right) \rightarrow H^{0}\left(U, \Omega_{M}^{3}\left(E_{M}\right)\right),
$$

is injective if the intersection of $U$ with every null line in $M$ is connected and an isomorphism if each such intersection is, moreover, simply connected.

It is sometimes convenient to place restrictions on the analytic cohomology of $U$ (e.g. that it be Stein) to obtain useful correspondences via the transform, which is no great loss of generality if one is interested primarily in local solutions of differential equations. However, the main concern of this paper is with the transform of bundles for which such assumptions are not necessary, and the restrictions to be placed on $U$ here relate to the topology of its intersection with the null lines in $M$. 
2.3. The field. Let $U, E_{A}, E_{M}$, etc., be as in $§ 2.2$. In this section the Yang-Mills field $F$ of $\left(E_{M}, \nabla\right)$ is described in terms of $E_{A}$ using the discussion of the extension problem given in $\$ 1.5$. Namely, for $x \in U$ it will be shown that $\left.E_{A}\right|_{Q_{x}^{(1)}}$ is trivial and $\mathbf{F}(x)$ corresponds to the difference between $\left.E_{A}\right|_{Q_{x}^{(2)}}$ and the trivial extension of $\left.E_{A}\right|_{Q_{x}^{(1)}}$ to $Q_{x}^{(2)}$, where $Q^{(n)}$ is the $n$th formal neighbourhood of $Q_{x} \hookrightarrow A$. This description is given in Manin [22].

Some essential cohomological calculations must first be dispensed with: suppose $x \in U$ corresponds to $L_{2} \subset T$. Let $I_{x} \subset \mathcal{O}_{A}$ and $K_{x} \subset \mathcal{O}_{P \times P^{*}}$ denote the sheaves of ideals defining $Q_{x}=\mathbb{P}\left(L_{2}\right) \times \mathbb{P}\left(T / L_{2}\right)$ as a closed subspace of $A$ and $P \times P^{*}$, respectively. Then

$$
K_{x} / K_{x}^{2}=\mathcal{O}\left(L_{2}\right)(-1,0) \oplus \mathcal{O}\left(T / L_{2}^{*}\right)(0,-1),
$$

and there is the exact sequence of conormal bundles

$$
0 \rightarrow J_{A} / J_{A}^{2} \otimes \mathcal{O} / K_{x} \rightarrow K_{x} / K_{x}^{2} \rightarrow I_{x} / I_{x}^{2} \rightarrow 0,
$$

with $J_{A} / J_{A}^{2} \otimes \mathcal{O} / K_{x}=\mathcal{O}_{Q_{x}}(-1,-1)$. Using Bott's Rule we find that

$$
H^{p}\left(Q_{x}, \mathcal{O}(-1,-1)\right)=0=H^{p}\left(Q_{x}, K_{x} / K_{x}^{2}\right) \text { for all } p,
$$

so $H^{p}\left(Q_{x}, I_{x} / I_{x}^{2}\right)=0$ for all $p$.

The second symmetric power of (2.3.1) gives the exact sequence

$$
0 \rightarrow K_{x} / K_{x}^{2} \otimes J_{A} / J_{A}^{2} \rightarrow K_{x}^{2} / K_{x}^{3} \rightarrow I_{x}^{2} / I_{x}^{3} \rightarrow 0 .
$$

Again using Bott's Rule, we obtain

$$
H^{p}\left(Q_{x}, K_{x} / K_{x}^{2}(-1,-1)\right)=0 \quad \text { for all } p, \quad H^{p}\left(Q_{x}, K_{x}^{2} / K_{x}^{3}\right)=0 \quad \text { for } p \neq 1,
$$

and

$$
\begin{aligned}
H^{1}\left(Q_{x}, K_{x}^{2} / K_{x}^{3}\right) & \simeq\left(\odot^{2} L_{2} \otimes \operatorname{det} L_{2}\right) \oplus\left(\odot^{2}\left(T / L_{2}\right)^{*} \otimes \operatorname{det}\left(T / L_{2}\right)^{*}\right) \\
& =\Omega_{M}^{2} \otimes \mathcal{O} / \mathbf{m}_{x} .
\end{aligned}
$$

Thus,

$$
H^{1}\left(Q_{x}, I_{x}^{2} / I_{x}^{3}\right) \simeq H^{1}\left(Q_{x}, K_{x}^{2} / K_{x}^{3}\right) \simeq \Omega_{M}^{2} \otimes \mathcal{O} / \mathbf{m}_{x} .
$$

Note that $a^{*}(2.3 .1)$ is the analytic restriction to $b^{-1}(x)$ of the exact sequence $0 \rightarrow J / J^{2} \otimes \mathcal{O} / K \rightarrow K / K^{2} \rightarrow I / I^{2} \rightarrow 0$ on $F$, so the preceding statements imply $b_{*}^{p} I / I^{2}=0$ for all $p, b_{*}^{p} I^{2} / I^{3}=0$ for $p \neq 1$, and $b_{*}^{1} K^{2} / K^{3} \simeq b_{*}^{1} I^{2} / I^{3} \simeq \Omega_{M}^{2}$.

Consider now the $Q$-trivial bundle $E_{A}$. By hypothesis, $\left.E_{A}\right|_{Q_{x}}$ is trivial, so $H^{1}\left(Q_{x}, \mathcal{O}\left(\right.\right.$ End $\left.\left.E_{A}\right) \otimes I_{x} / I_{x}^{2}\right)=0$. Since this group acts transitively on $\Sigma_{1}\left(\left.E_{A}\right|_{Q_{x}}\right)$, it Follows that $\left.E_{A}\right|_{Q_{x}^{(1)}}$ is the unique and, therefore, trivial extension of $\left.E_{A}\right|_{Q_{x}}$ to $Q_{x}^{(1)}$.

Since there is, in particular, the trivial extension of $\left.E_{A}\right|_{Q_{x}^{(1)}}$ to $Q_{x}^{(2)}$, the action of $H^{1}\left(Q_{x}, \mathcal{O}\left(\right.\right.$ End $\left.\left.E_{A}\right) \otimes I_{x}^{2} / I_{x}^{3}\right)$ on $\Sigma_{2}\left(\left.E_{A}\right|_{Q_{x}^{(1)}}\right)$ is effective as well as transitive, so there is a unique element of this vector space representing the difference between $\left.E_{A}\right|_{Q_{x}^{(2)}}$ and the trivial extension of $\left.E_{A}\right|_{Q_{x}^{(1)}}$ to $Q_{x}^{(2)}$. It will shortly be seen that this element corresponds to the value of the field at $x$ under the canonical isomorphism

$$
H^{1}\left(Q_{2}, \mathcal{O}\left(\text { End } E_{A}\right) \otimes I_{x}^{2} / I_{x}^{3}\right) \simeq \Omega_{M}^{2}\left(\text { End } E_{M}\right) \otimes \mathcal{O} / \mathbf{m}_{x} .
$$


Let $F^{(n)}$ denote the $n$th formal neighbourhood of $F \hookrightarrow M \times A$, and let $a^{(n)}$ : $F^{(n)} \rightarrow A, b^{(n)}: F^{(n)} \rightarrow M$ be the restrictions to $F^{(n)}$ of the projections. Let $U^{\prime(n)}:=\left(U^{\prime},\left.\mathcal{O}_{F^{(n)}}\right|_{U^{\prime}}\right)$ and, as in $\S 2.2$, let $a^{(n)}$ and $b^{(n)}$ denote the restrictions to $U^{(n)}$ of the appropriate maps when the underlying set $U$ is understood.

The global analogue of the preceding analysis is as follows: let $E_{F^{(n)}}:=a^{(n)^{*}} E_{A}$ (with $E_{F}=E_{F^{(0)}}$ as before). Since $H^{1}\left(U^{\prime}, \mathcal{O}\left(\right.\right.$ End $\left.\left.E_{F}\right) \otimes I / I^{2}\right)=0$ and this group acts transitively on $\Sigma_{1}\left(E_{F}\right)$, it follows that $E_{F^{(1)}}$ is the unique extension of $E_{F}$ to $U^{\prime(1)}$, and, therefore, $b^{(1)^{*}} E_{M} \simeq E_{F^{(1)}}$. In fact, since $H^{0}\left(U^{\prime}, \mathcal{O}\left(\right.\right.$ End $\left.\left.E_{F}\right) \otimes I / I^{2}\right)$ also vanishes, there is a unique extension of the isomorphism $b^{*} E_{M} \simeq a^{*} E_{A}$ to $U^{\prime(1)}$.

Since

$$
\Gamma\left(U^{\prime}, \mathcal{O}\left(\text { End } b^{(2)^{*}} E_{M}\right)\right) \rightarrow \Gamma\left(U^{\prime}, \mathcal{O}\left(\text { End } E_{F^{(1)}}\right)\right)
$$

is surjective, $H^{1}\left(U^{\prime}, \mathcal{O}\right.$ (End $\left.\left.E_{F}\right) \otimes I^{2} / I^{3}\right)$ acts effectively as well as transitively on $\Sigma_{2}\left(E_{F^{(1)}}\right)$, so there is a unique element of this group representing the difference between $b^{(2)^{*}} E_{M}$ and $E_{F^{(2)}}$, and the global problem is to show that this element corresponds to the field $\mathbf{F}(\nabla)$ under the isomorphism

$$
H^{1}\left(U^{\prime}, \mathcal{O}\left(\text { End } E_{F}\right) \otimes I^{2} / I^{3}\right) \simeq H^{0}\left(U, \Omega_{M}^{2}\left(\text { End } E_{M}\right)\right) \text {. }
$$

To see this, let $\nabla_{a^{(n)}}$ denote the relative connection on $b^{(n)^{*}} E_{M}$ induced by $\nabla$ on $E_{M}$. Then by the relative version of Proposition $\mathrm{H}(\mathrm{a})$, there is a unique isomorphism $\left(b^{(1)^{*}} E_{M}, \tilde{\nabla}_{a}\right) \simeq\left(E_{F^{(1)}}, \tilde{d}_{a}\right)$ restricting to the identity on $\left(E_{F}, d_{a}\right)$. Since $\Omega_{a}^{2}=0$, (1.3.4) in this case reads

$$
0 \rightarrow I^{2} / I^{3} \rightarrow \Omega_{M}^{1} \otimes I / I^{2} \rightarrow \Omega_{M}^{2} \otimes \mathcal{O} / I \rightarrow 0
$$

and $R_{a}^{2} \simeq \Omega_{M}^{2} \otimes \mathcal{O} / I$. The relative version of Proposition $\mathrm{H}(\mathrm{c})$ then states that $\nabla_{a^{(1)}}-d_{d^{(1)}} \in H^{0}\left(U^{\prime}, \Omega_{M}^{2}\left(\right.\right.$ End $\left.\left.E_{F}\right)\right)$ is just

$$
\left.\left(\mathbf{F}\left(\nabla_{a^{(1)}}\right)-\mathbf{F}\left(d_{a^{(1)}}\right)\right)\right|_{U^{\prime}}=b^{*} \mathbf{F}(\nabla)
$$

and its image in $H^{1}\left(U^{\prime}, \mathcal{O}\left(\right.\right.$ End $\left.\left.E_{F}\right) \otimes I^{2} / I^{3}\right)$ under the connecting map from (2.3.2) $\otimes \mathcal{O}\left(\right.$ End $\left.E_{F}\right)$ is the difference between the two extensions of $E_{F^{(1)}}$ to $U^{\prime(2)}$ determined by $\nabla_{a^{(1)}}$ and $d_{a^{(1)}}$; this must be $b^{(2)^{*}} E_{M}-E_{F^{(2)}}$ by the uniqueness statement of $\mathrm{H}(\mathrm{a})$. This proves the assertion.

(Although the vanishing of $b_{*}^{q} I / I^{2}$ for all $q$ does not appear to have been used here and merely contributes the information that the connecting map of (2.3.2) $\otimes$ $\mathcal{O}\left(\right.$ End $\left.E_{F}\right)$ is an isomorphism, this fact has been implicitly used to construct the connection on $E_{M}$.)

Note that the map

$$
\mathcal{O}\left(E_{M}\right)=b_{*} \mathcal{O}\left(E_{F^{(1)}}\right) \rightarrow b_{*}^{1} \mathcal{O}\left(E_{F}\right) \otimes I^{2} / I^{3}=\Omega_{M}^{2}\left(E_{M}\right)
$$

induced from

$$
0 \rightarrow \mathcal{O}\left(E_{F}\right) \otimes I^{2} / I^{3} \rightarrow \mathcal{O}\left(E_{F^{(2)}}\right) \rightarrow \mathcal{O}\left(E_{F^{(1)}}\right) \rightarrow 0
$$

is just $-\nabla^{2}: \mathcal{O}\left(E_{M}\right) \rightarrow \Omega^{2}\left(E_{M}\right)$.

Although the field does not correspond precisely to any global cohomology class on $U^{\prime \prime}$ in the sense of the transform described in $\$ 2.2$, it is nevertheless derived from one-namely, $-D E_{A} \in H^{1}\left(U^{\prime \prime}, \Omega_{A}^{1}\left(\right.\right.$ End $\left.\left.E_{A}\right)\right)$. If $D E_{A}$ is pulled back to $U^{\prime(1)}$, its 
image in $H^{1}\left(U^{\prime}, \Omega_{b^{(1)}}^{1}\left(\right.\right.$ End $\left.E_{\left.F^{(1)}\right)}\right)$ must be zero since $E_{F^{(1)}} \simeq b^{(1)^{*}} E_{M}$. From the exact sequence $0 \rightarrow I^{2} / I^{3} \rightarrow \Omega_{A}^{1} \otimes \mathcal{O} / I^{2} \rightarrow \Omega_{b^{(1)}}^{1} \rightarrow 0$ and the fact that $b_{*} \Omega_{b^{(1)}}^{1}=0$, it follows that there is a unique class in $H^{1}\left(U^{\prime}, \mathcal{O}\left(\right.\right.$ End $\left.\left.E_{F}\right) \otimes I^{2} / I^{3}\right)$ mapped to $a^{(1)^{*}} D E_{A}$ and this, by the arguments of $\S 1.5$, is precisely $E_{F^{(2)}}-b^{(2)^{*}} E_{M}$. In fact, a similar argument shows that $\mathbf{F}_{+} \in H^{0}\left(U, \Omega_{+}^{2}\left(\right.\right.$ End $\left.\left.E_{M}\right)\right)$ corresponds in this sense to $-D_{c} E_{A} \in H^{1}\left(U^{\prime \prime}, \Omega_{c}^{1}\left(\right.\right.$ End $\left.\left.E_{A}\right)\right)$, where $c: A \rightarrow P^{*}$ is the "projection". Of course, the analogous result for $\mathbf{F}_{-}$is also true.

If the field vanishes identically and the fibres of $a$ are connected, then by Proposition $\mathrm{G}$ the flat connection on $E_{F}$ pushes down to a connection on $E_{A}$ which is necessarily flat. This is nothing more than the obvious statement that the construction gives a map $H^{1}\left(U^{\prime \prime}, \mathrm{GL}(N, \mathbb{C})\right) \rightarrow H^{1}(U, \mathrm{GL}(N, \mathbb{C}))$ which is injective when the fibres of $a$ are connected and bijective when they are, moreover, simply connected.

2.4. The current. Let $A^{(n)}$ be the $n$th formal neighbourhood of $A$ in $P \times P^{*}$, and let $U^{\prime \prime}:=\left(U^{\prime \prime},\left.\mathcal{O}_{A^{(n)}}\right|_{U^{\prime \prime}}\right)$. In this section using the formalism developed in Part I, the main results of Manin and Witten, Isenberg, Yasskin, and Green are presented. These results are: if the intersection of $U$ with every null line in $M$ is connected and simply connected, then (a) $E_{A}$ extends (nonuniquely) to $U^{\prime \prime(1)}$, (b) there is a unique such extension $E_{A^{(1)}}$ admitting a further (unique) extension to a bundle $E_{A^{(2)}}$ on $U^{\prime \prime(2)}$, and (c) the obstruction $\omega_{3}\left(E_{A^{(2)}}\right)$ to extending $E_{A^{(2)}}$ to $U^{\prime \prime(3)}$ corresponds to the Yang-Mills current $\mathbf{J}$ under the transform for cohomology (2.2.1):

$$
\begin{array}{ccccc}
H^{2}\left(U^{\prime \prime}, \mathcal{O}\left(\text { End } E_{A}\right) \otimes \frac{J_{A}^{3}}{J_{A}^{4}}\right) & \rightarrow & {\left[\operatorname{ker} \nabla: H^{0}\left(U, \Omega_{M}^{3}\left(\text { End } E_{M}\right)\right)\right.} & \rightarrow & \left.H^{0}\left(U, \Omega_{M}^{4}\left(\text { End } E_{M}\right)\right)\right] \\
\ni & & \ni \\
\omega_{3}\left(E_{A^{(2)}}\right) & \rightarrow & -\frac{1}{12} \mathbf{J}
\end{array}
$$

The notation used here is that of $\S 1.3: F^{[n]}$ is the $n$th formal neighbourhood of $F$ in $M \times P \times P^{*}$, and $F^{(n, m)}$ is the $m$ th formal neighbourhood of $F^{(n)}$ in $F^{[n]}$-recall that $F^{(n, m)}$ is defined by $K^{n+1}+J^{m+1} \subset \mathcal{O}_{M \times P \times P^{*}}$ as a closed subspace of $M \times P$ $\times P^{*}$. Further, $a^{(n, m)}: F^{(n, m)} \rightarrow A^{(m)}$ and $b^{(n, m)}: F^{(n, m)} \rightarrow M$ denote the restrictions to $F^{(n, m)}$ of the projections, and the usual convention that these symbols also denote their restrictions to $U^{(n, m)}:=\left(U^{\prime},\left.\mathcal{O}_{F^{(n, m)}}\right|_{U^{\prime}}\right)$ applies. We assume throughout that the intersection of $U$ with every null line in $M$ is connected and simply connected.

By Proposition $F$ the extension problem for $E_{A}$ to $U^{\prime \prime(n)}$ is equivalent to the extension problem for $\left(E_{F^{(n)}}, d_{a^{(n)}}\right)$ to $U^{\prime[n]}$. By working entirely on $U^{\prime}$ and its formal neighbourhoods, the cohomological calculations are less complicated, and the ability to treat the extension problem for the bundle and the flat relative connection separately turns out to be a useful simplification. A judicious choice of path up through the formal neighbourhoods of $F$ "solves" the extension problem for the bundle in an economical way, and the "solution" of the extension problem for the relative connection can be read off from the exact sequences (1.3.12) and (1.3.13). In this context (1.3.13), with $q=n$ and $m=0$, is particularly useful and, in the current 
notation, is

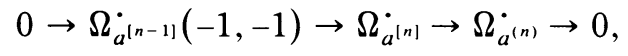

which is a resolution of the topological inverse image of $0 \rightarrow J_{A} / J_{A}^{n+1} \rightarrow \mathcal{O}_{A^{(n)}} \rightarrow \mathcal{O}_{A}$ $\rightarrow 0$.

Consider, first, extensions to $U^{\prime[1]}$ :

$$
H^{1}\left(U^{\prime}, \mathcal{O}\left(\text { End } E_{F}\right)(-1,-1)\right)=0=H^{1}\left(U^{\prime}, \mathcal{O}\left(\text { End } E_{F}\right) \otimes K / K^{2}\right),
$$

and, therefore, $b^{[1]^{*}} E_{M}:=E_{F^{[1]}}$ is the unique extension to $U^{\prime[1]}$ of both $E_{F}$ and $E_{F^{(1)} \text {. }}$

From $(2.4 .1) \otimes \mathcal{O}\left(\right.$ End $\left.E_{F^{(1)}}\right)$,

$$
\omega_{1}\left(d_{a^{(1)}}\right) \in H^{1}\left(U^{\prime}, \Omega_{a}^{1}\left(\text { End } E_{F}\right)(-1,-1)\right),
$$

a group which does not necessarily vanish, since it is isomorphic to $H^{1}\left(U, \mathcal{O}\left(\right.\right.$ End $\left.\left.E_{M}\right)[-1][-1]^{\prime}\right)$. However, it will shortly be seen that $d_{a^{(1)}}$ has at least one extension to $E_{F^{(1)}}$ and, granted this, $\Sigma_{1}\left(d_{a^{(1)}}\right)$ is in one-to-one correspondence with

$$
H^{0}\left(U^{\prime}, \Omega_{a}^{1}\left(\text { End } E_{F}\right)(-1,-1)\right) \simeq H^{0}\left(U, \mathcal{O}\left(\text { End } E_{M}\right)[-1][-1]^{\prime}\right) .
$$

Since $\Omega_{a}^{2}=0$, each such extension is flat, so by Proposition $\mathrm{F}$ there is a unique extension of $E_{A}$ to $U^{\prime \prime(1)}$ for each choice of extension of $d_{a^{(1)}}$.

Note that $\tilde{d}_{a}: \mathcal{O}\left(E_{F^{(1)}}\right) \rightarrow \Omega_{M}^{1}\left(E_{F}\right)$ extends uniquely to a map $\tilde{\nabla}_{a}: \mathcal{O}\left(E_{F^{(1)}}\right) \rightarrow$ $\Omega_{M}^{1}\left(E_{F}\right)$, so this must be the same as the map induced by $\nabla$ on $E_{M}$.

Consider now the extensions of $E_{F^{[1]}}$ to $U^{[2]}$ : since $E_{F^{[1]}}=b^{[1]^{*}} E_{M}, E_{F^{[1]}}$ certainly extends. The surjectivity of $H^{0}\left(U^{\prime}, \mathcal{O}\left(\right.\right.$ End $\left.\left.b^{[2]^{*}} E_{M}\right)\right) \rightarrow H^{0}\left(U^{\prime}, \mathcal{O}\left(\right.\right.$ End $\left.\left.E_{F^{[1]}}\right)\right)$ implies that the action of $H^{1}\left(U^{\prime}, \mathcal{O}\left(\right.\right.$ End $\left.\left.E_{F}\right) \otimes K^{2} / K^{3}\right)$ on $\Sigma_{2}\left(E_{F^{[1]}}\right)$ is both transitive and effective, and, since

$$
H^{1}\left(U^{\prime}, \mathcal{O}\left(\text { End } E_{F}\right) \otimes K^{2} / K^{3}\right) \rightarrow H^{1}\left(U^{\prime}, \mathcal{O}\left(\text { End } E_{F}\right) \otimes I^{2} / I^{3}\right)
$$

is an isomorphism, it follows that there is a unique extension $E_{F^{[2]}}$ of $E_{F^{[1]}}$ to $U^{[2]}$ satisfying $\left.E_{F^{(2)}}\right|_{U^{\prime(2)}}=E_{F^{(2)}}$.

By direct calculation, $b_{*}^{q} \Omega_{a^{111}}^{p_{1}}(-1,-1)=0$ for all $p$ and $q$, so from $(2.4 .1) \otimes$ $\mathcal{O}\left(\right.$ End $\left.E_{F^{[2]}}\right)$ it follows that $d_{a^{(2)}}$ extends to a unique and flat relative connection $\nabla_{a^{[2]}}$ on $E_{F^{(2)}}$. This validates the claim concerning the extension of $d_{a^{(1)}}$, and, by Proposition $\mathrm{F}$ again, one now obtains a unique extension $E_{A^{(2)}}$ of $E_{A}$ to $U^{\prime \prime(2)}$ satisfying $\left(a^{[2]^{*}} E_{A^{(2)}}, d^{[2]}\right) \simeq\left(E_{F^{[2]}}, \nabla_{a^{[2]}}\right)$.

Note that the field $\mathbf{F}$ also corresponds to $b^{[2]^{*}} E_{M}-E_{F^{[2]}}$ under the isomorphism

$$
\begin{aligned}
H^{1}\left(U^{\prime}, \mathcal{O}\left(\text { End } E_{F}\right) \otimes K^{2} / K^{3}\right) & \sim H^{1}\left(U^{\prime}, \mathcal{O}\left(\text { End } E_{F}\right) \otimes I^{2} / I^{3}\right) \\
& \simeq H^{0}\left(U, \Omega_{M}^{2}\left(\text { End } E_{M}\right)\right) .
\end{aligned}
$$

Finally, the case of extensions to $U^{\prime[3]}$ : this case is more difficult because $E_{F^{[2]}} \neq b^{[2]^{*}} E_{M}$, in general, and the direct images are accordingly harder to deal with. The extension problem itself is simply that of extending $E_{F^{(3,2)}}:=a^{(3,2)^{*}} E_{A^{(2)}}$ and $d_{a^{(3.2)}}$ to $U^{\prime[3]}$; the difficult part is showing that $\omega_{3}\left(E_{F^{(3.2)}}\right)\left(=a^{*} \omega_{3}\left(E_{A^{(2)}}\right)\right)$ does indeed correspond to the current $\mathbf{J}$. 
The problem for the relative connection is easily "solved": since $H^{p}\left(U^{\prime}, \Omega_{a}^{q}\left(\right.\right.$ End $\left.\left.E_{F}\right)(-3,-3)\right)=0$ for $p, q=0,1$, any extension $E_{F^{[3]}}$ of $E_{F^{(3,2)}}$ to $U^{\prime 3]}$ is unique and, from $(1.3 .12) \otimes \mathcal{O}\left(\right.$ End $\left.E_{F^{(3)}}\right), d_{a^{(3,2)}}$ extends to a unique and flat relative connection on $E_{F^{[3]}}$.

To reduce the problem to diagram-chasing on $U^{\prime[1]}$ rather than $U^{{ }^{[2]}}$, the first step is to extend $E_{F^{[2]}}$ to $U^{\prime[3]}$. If one could obtain such an extension $E_{F^{[3]}}$, then

$$
E_{F^{(3.2)}}-\left.E_{F^{(3)}}\right|_{U^{\prime(3.2)}} \in H^{1}\left(U^{\prime}, \mathcal{O}\left(\text { End } E_{F}\right) \otimes K^{3} / K^{4}+J^{3}\right)
$$

and the image of this class under the connecting homomorphism from

$$
0 \rightarrow J^{3} / J^{4} \otimes \mathcal{O} / K \rightarrow K^{3} / K^{4} \rightarrow K^{3} / K^{4}+J^{3} \rightarrow 0
$$

is precisely $\omega_{3}\left(E_{F^{(3,2)}}\right) \in H^{2}\left(U^{\prime}, \mathcal{O}\left(\right.\right.$ End $\left.\left.E_{F}\right) \otimes J^{3} / J^{4}\right)$.

To see that $E_{F^{[2]}}$ does extend to $U^{\prime[3]}$, one takes direct images of (2.4.2): the direct images of the first two sheaves are easily calculated using Bott's Rule. The direct images of $K^{3} / K^{4}+J^{3}$ are best evaluated using the complex

$$
0 \rightarrow K^{3} / K^{4}+J^{3} \rightarrow \Omega_{M}^{1} \otimes K^{2} / K^{3} \rightarrow \Omega_{M}^{2} \otimes K / K^{2} \rightarrow \Omega_{M}^{3} \otimes \mathcal{O} / K \rightarrow 0 .
$$

As noted in $\S 1.3$, the cohomology of this complex is 0 except at $\Omega_{M}^{1} \otimes K^{2} / K^{3}$, at which term the cohomology is $\Omega_{a}^{1} \otimes J^{2} / J^{3}=\mathcal{O}_{F}(-1,-1)[-1][-1]^{\prime}$. Since $b_{*}^{q} \mathcal{O}_{F}(-1,-1)=0$ for all $q,(2.4 .3)$ can be regarded as exact when taking direct images, thus leading to the identification of $b_{*}^{q} K^{3} / K^{4}+J^{3}$ as 0 for $q \neq 1$, and

$$
b_{*}^{1} K^{3} / K^{4}+J^{3}=\operatorname{ker} \Lambda: \Omega_{M}^{1} \otimes \Omega_{M}^{2} \rightarrow \Omega_{M}^{3}
$$

(where $\Lambda$ is the map induced by $\Omega_{M}^{1} \otimes \Omega_{M}^{2} \ni f \otimes g \rightarrow f \wedge g \in \Omega_{M}^{3}$ ).

The direct image of (2.4.2) is the following exact sequence on $M$ :

$$
\begin{aligned}
& 0 \rightarrow \quad b_{*}^{1} K^{3} / K^{4} \rightarrow \begin{array}{c}
b_{*}^{1} K^{3} / K^{4}+J^{3} \\
\|
\end{array} \quad b_{*}^{2} J^{3} / J^{4} \otimes \mathcal{O} / K \quad \rightarrow \quad 0
\end{aligned}
$$

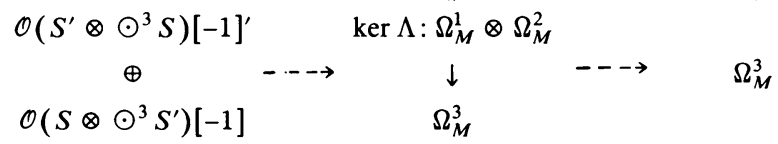

The right-hand dashed arrow is the map induced by $\Omega_{M}^{1} \otimes \Omega_{M}^{2} \ni f \otimes g \mapsto$ $-\frac{1}{12} f \wedge * g \in \Omega_{M}^{3}$, so this sequence is split globally on $M$ by the composition

$$
\Omega_{M}^{3} \stackrel{\text { inc }}{\rightarrow} \Omega_{M}^{1} \otimes \Omega_{M}^{2} \stackrel{-12 \text { id }}{\rightarrow}{ }^{*} \Omega_{M}^{1} \otimes \Omega_{M}^{2} .
$$

All other direct images of the sheaves in (2.4.2) vanish, so

$$
H^{2}\left(U^{\prime}, \mathcal{O}\left(\text { End } E_{F}\right) \otimes K^{3} / K^{4}\right) \rightarrow H^{2}\left(U^{\prime}, \mathcal{O}\left(\text { End } E_{F}\right) \otimes K^{3} / K^{4}+J^{3}\right)
$$

is injective, and, since $\omega_{3}\left(E_{F^{(2)}}\right)$ is sent to zero by this map, it follows that $\omega_{3}\left(E_{F^{[2]}}\right)=0$ as claimed.

Let $E_{F^{[3]}}$ be any extension of $E_{F^{[2]}}$ to $U^{\prime[3]}$; then, since $b^{[3]^{*}} E_{M}$ is also an extension of $E_{F^{[1]}}$ to $U^{\prime[3]}$, one has the (unique) class $b^{[3]^{*}} E_{M}-E_{F^{[3]}}$ in

$$
H^{1}\left(U^{\prime}, \mathcal{O}\left(\text { End } E_{F^{[1]}}\right) \otimes K^{2} / K^{4}\right) \text {. }
$$


( $F^{[3]}$ is the first formal neighbourhood of $F^{[1]}$ in $M \times P \times P^{*}$.) The image of $b^{[3]^{*}} E_{M}-E_{F^{[3]}}$ in $H^{1}\left(U^{\prime}, \mathcal{O}\left(\right.\right.$ End $\left.\left.E_{F^{[1]}}\right) \otimes K^{2} / K^{4}+J^{3}\right)$ is the sum of

$$
b^{(3,2)^{*}} E_{M}-E_{F^{(3,2)}} \in H^{1}\left(U^{\prime}, \mathcal{O}\left(\text { End } E_{F^{(1)}}\right) \otimes K^{2} / K^{4}+J^{3}\right)
$$

and

$$
E_{F^{(3.2)}}-\left.E_{F^{(3)}}\right|_{U^{\prime 3.2)}} \in H^{1}\left(U^{\prime}, \mathcal{O}\left(\text { End } E_{F}\right) \otimes K^{3} / K^{4}+J^{3}\right) .
$$

On tensoring through the diagram

$$
\begin{aligned}
& 0 \rightarrow \Omega_{M}^{1} \otimes K / K^{3} \rightarrow \Omega_{M}^{1} \otimes \mathcal{O} / K^{3} \rightarrow \Omega_{M}^{1} \otimes \mathcal{O} / K \quad \rightarrow \quad 0 \\
& 0 \rightarrow K^{2} / K^{4}+J^{3} \rightarrow \mathcal{O} / K^{4}+J^{3} \rightarrow \quad \begin{array}{c}
\uparrow \\
0 / K^{2}
\end{array} \rightarrow 0
\end{aligned}
$$

by $\operatorname{Hom}\left(E_{F^{(3.2)}}, b^{(3,2)^{*}} E_{M}\right)$ and altering the vertical maps to those induced by $d_{a^{(3.2)}}$ on $E_{F^{(3.2)}}$ and $\nabla$ on $E_{M}$, one sees that the image of $b^{(3,2)^{*}} E_{M}-E_{F^{(3.2)}}$ in

$$
H^{1}\left(U^{\prime}, \Omega_{M}^{1}\left(\text { End } E_{F^{[1]}}\right) \otimes K / K^{3}\right)
$$

is zero, since the image of the identity endormorphism of $E_{F^{(1)}}$ in $H^{0}\left(U^{\prime}, \Omega_{M}^{1}\left(\right.\right.$ End $\left.\left.E_{F}\right)\right)$ is zero.

Consider now the diagram

$$
\begin{aligned}
& \begin{array}{ccccccc}
0 & \rightarrow & K^{3} / K^{4}+J^{3} & \rightarrow & \Omega_{M}^{1} \otimes K / K^{3} & \rightarrow & X \\
\uparrow & & \uparrow & & \uparrow & &
\end{array} \\
& 0 \rightarrow K^{3} / K^{4} \rightarrow K^{2} / K^{4} \rightarrow K^{2} / K^{3} \rightarrow 0
\end{aligned}
$$

where $X$ is defined by the upper row, and the right-hand vertical arrow is the induced map. Tensoring through by $\operatorname{Hom}\left(E_{F^{[1]}}, b^{[1]^{*}} E_{M}\right)$ and altering the vertical maps to those induced by $d_{a^{[1]}}$ on $E_{F^{(1)}}$ and $\nabla$ on $E_{M}$, one has the following:

$$
b^{[2]^{*}} E_{M}-E_{F^{[2]}} \in H^{1}\left(U^{\prime}, \mathcal{O}\left(\text { End } E_{F}\right) \otimes K^{2} / K^{3}\right)
$$

pulls back to

$$
b^{[3]^{*}} E_{M}-E_{F^{[3]}} \in H^{1}\left(U, \mathcal{O}\left(\text { End } E_{F^{[1]}}\right) \otimes K^{2} / K^{4}\right) ;
$$

the image of the latter class in $H^{1}\left(U^{\prime}, \Omega_{M}^{1}\left(\right.\right.$ End $\left.\left.E_{F^{11]}}\right) \otimes K / K^{3}\right)$ is the sum of the images of $b^{(3,2)^{*}} E_{M}-E_{F^{(3,2)}}$ and $E_{F^{(3,2)}}-\left.E_{F^{(3)}}\right|_{F^{(3,2)}}$, the former of which has just been shown to vanish; finally, the image of $E_{F^{(3.2)}}-\left.E_{F^{(3)}}\right|_{F^{(3.2)}}$ in

$$
H^{2}\left(U^{\prime}, \mathcal{O}\left(\text { End } E_{F}\right) \otimes J^{3} / J^{4}\right)
$$

under the connecting homomorphism from $(2.4 .2) \otimes \mathcal{O}\left(\right.$ End $\left.E_{F}\right)$ is $\omega_{3}\left(E_{F^{(3.2)}}\right)=$ $a^{*} \omega_{3}\left(E_{A^{(2)}}\right)$.

From its definition, $b_{*}^{1} X=\Omega_{M}^{3}$, with all other direct images vanishing. Hence, the rows of $(2.4 .5)$ remain exact on application of the functor $b_{*}^{1}$, and the resulting diagram induces a connecting map

$$
\begin{aligned}
& {\left[\operatorname{ker} b_{*}^{1} K^{2} / K^{3} \rightarrow b_{*}^{1} X\right] \rightarrow\left[\operatorname{coker} b_{*}^{1} K^{3} / K^{4} \rightarrow b_{*}^{1} K^{3} / K^{4}+J^{3}\right]} \\
& \text { II } \\
& \|\quad\| \quad b_{*}^{2} J^{3} / J^{4} \otimes \mathcal{O} / K \\
& \Omega_{M}^{2} \quad \rightarrow \quad \Omega_{M}^{3} \quad \cdots \rightarrow \quad \Omega_{M}^{3} \text {. }
\end{aligned}
$$


Using the identifications of the induced maps in (2.4.4), the dashed arrow here is just $-\frac{1}{12} d_{*}:\left[\operatorname{ker} d: \Omega_{M}^{2} \rightarrow \Omega_{M}^{3}\right] \rightarrow \Omega_{M}^{3}$; therefore, the image of $\omega_{3}\left(E_{A^{(2)}}\right)$ in $H^{0}\left(U, \Omega_{M}^{3}\left(\right.\right.$ End $\left.\left.E_{M}\right)\right)$ is $-\frac{1}{12} \nabla * \mathbf{F}=-\frac{1}{12} \mathbf{J}$; this completes the proof.

The introduction of $E_{F^{(3)}}$ is to some extent redundant in that $\omega_{3}\left(E_{F^{(3,2)}}\right)$ is the image of

$$
E_{F^{(3,2)}}-b^{(3,2)^{*}} E_{M} \in H^{1}\left(U^{\prime},\left(\text { End } E_{F^{[1]}}\right) \otimes K^{2} / K^{4}+J^{3}\right)
$$

under the connecting homomorphism from

$$
0 \rightarrow J^{3} / J^{4} \otimes \mathcal{O} / K \rightarrow K^{2} / K^{4} \rightarrow K^{2} / K^{4}+J^{3} \rightarrow 0,
$$

but the problem of identifying the relevant induced maps on $M$ is more complicated without this. Note also that $E_{F^{(3,2)}}-\left.E_{F^{(3)}}\right|_{F^{(3.2)}}$ is also the obstruction to extending $d_{a^{[2]}}$ on $E_{F^{[2]}}$ to a map $\mathcal{O}\left(E_{F^{[3]}}\right) \rightarrow \Omega_{M}^{1}\left(E_{F^{[2]}}\right)$.

2.5. Higher order obstructions. With the same notation, conventions, and objects of the previous section, suppose that $E_{A}$ has been extended to a bundle $E_{A^{(n)}}$ for some $n \geqslant 2$. The purpose of this section is to outline the identification of the class in

$$
H^{0}\left(U, \mathcal{O}\left(\odot^{n-1} S \otimes \odot^{n-1} S^{\prime}\right)\left(\text { End } E_{M}\right)[-1][-1]^{\prime}\right)
$$

corresponding to

$$
\omega_{n+1}\left(E_{A^{(n)}}\right) \in H^{2}\left(U^{\prime \prime}, \mathcal{O}\left(\text { End } E_{A}\right) \otimes J_{A}^{n+1} / J_{A}^{n+2}\right)
$$

under the transform for cohomology.

First, some preliminary observations: the transform gives

$$
H^{1}\left(U^{\prime \prime}, \mathcal{O}\left(\text { End } E_{A}\right) \otimes J_{A}^{n+1} / J_{A}^{n+2}\right)=0 \quad(\text { for } n \geqslant 1),
$$

so any extension of $E_{A^{(n)}}$ to $U^{\prime \prime(n+1)}$ is unique if it exists. When $n \geqslant 3$,

$$
b_{*}^{2} J^{n+1} / J^{n+2} \otimes \mathcal{O} / K \rightarrow b_{*}^{2} K^{n+1} / K^{n+2}
$$

is injective, so, in contrast with the case $n=2, E_{A^{(n)}}$ extends iff $E_{F^{(n)}}:=a^{[n]^{*}} E_{A^{(n)}}$ extends to $U^{\prime[n+1]}$ (if $\omega_{n+1}\left(E_{F^{[n]}}\right)=0$, there is a unique extension of $E_{F^{[n]}}$ to $U^{\prime[n+1]}$ admitting a (flat) extension of $\left.d_{a^{[n]}}\right)$. For purposes of identifying the obstruction, however, this observation turns out to be not particularly useful, and a different approach is required.

This approach repeats the complete transform procedure, but with diagram (2.1.1) replaced by

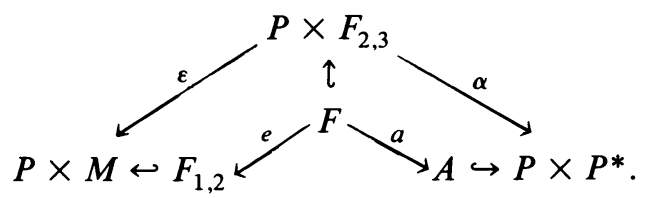

With this picture the derived correspondence is between $Q$-trivial bundles on $A^{(n)}$ and bundles on $F_{1,2}^{(n)}$ which admit "half-flat" relative connections along the fibres of $F_{1,2}^{(n)} \rightarrow P$ - these terms will be made precise shortly. In what follows, each unproved assertion can be proved in precisely the same way as the analogous statement in $\S \S 2.3$ or 2.4 . 
If $F^{\{n\}}$ denotes the $n$th formal neighbourhood of $F$ in $P \times F_{2,3}$, then, by Proposition $\mathrm{F}$, the extension problem for $E_{A^{(n)}}$ to $U^{\prime \prime(n+1)}$ is equivalent to that for $\left(a^{\{n\}^{*}} E_{A^{(n)}}, d_{a^{(n)}}\right)$ to $U^{\{n+1\}}$. Just as with $E_{F^{(2)}}, E_{F^{\{n\}}}:=a^{\{n\}^{*}} E_{A^{(n)}}$ always extends to $U^{\prime\{n+1\}}$, and $\omega_{n+1}\left(E_{A^{(n)}}\right)$ is now manifested as the obstruction $\tilde{\omega}_{n+1}\left(d_{a^{(n\}}}\right)$ to extending $d_{a^{(n)}}$ to a map $\tilde{\nabla}_{a^{(n)}}: \mathcal{O}\left(E_{F^{(n+1)}}\right) \rightarrow \Omega_{\alpha}^{1}\left(E_{F^{\{n\}}}\right)$ (modulo choice of $E_{F^{\{n+1\}}} \in$ $\left.\sum_{n+1}\left(E_{F^{\{n\}}}\right)\right)$.

The bundle $E_{F^{\{n\}}}$ admits a unique and flat relative connection $\nabla_{e^{\{n\}}}$ and thus pushes down to $U_{1}^{(n)}$, where $U_{1}^{\prime}:=e\left(U^{\prime}\right)$. Alternatively, $e_{*}\left(E_{F^{(n)}}\right)=: \mathcal{O}\left(E_{F_{1,2}^{(n)}}\right)$ is locally free and $e^{\{n\}^{*}} E_{F_{1,2}^{(n)}}=E_{F^{(n)}}$. As with the construction of $E_{M}, E_{F_{1,2}^{(n)}}$ has some extra structure induced by $d_{a^{\{n\}}}$-this time it is a map

$$
\tilde{\nabla}_{f^{(n-1)}}: \mathcal{O}\left(E_{F_{1,2}^{(n)}}\right) \rightarrow \Omega_{M}^{1}\left(E_{F_{1,2}^{(n-1)}}\right), \text { where } f^{(n)}: F_{1,2}^{(n)} \rightarrow P .
$$

Since $d_{a^{(n)}}$ is flat, $\tilde{\nabla}_{f^{(n-1)}}$ is half-flat in the sense that the composition $\mathcal{O}\left(E_{F_{1.2}^{(n)}}\right) \rightarrow$ $\Omega_{M}^{1}\left(E_{F_{1,2}^{(n-1)}}\right) \rightarrow \Omega_{+}^{2}\left(E_{F_{1,2}^{(n-2)}}\right)$ is zero. $\left(\Omega_{+}^{2}\right.$ arises from the fact that $e_{*} \Omega_{\alpha}^{2} \otimes \mathcal{O}_{F^{\{n\}}}=\Omega_{+}^{2}$ $\otimes \mathcal{O}_{F_{1,2}^{(n)}} ;$ this is, of course, the wrong half of the curvature to vanish to induce a flat relative connection on $E_{F_{1,2}}$ )

If $I_{1} \subset \mathcal{O}_{P \times M}$ defines $F_{1,2} \hookrightarrow P \times M$, and $g: F_{1,2} \rightarrow M$ is the projection, then $g^{(1)^{*}} E_{M} \simeq E_{F_{1,2}^{(1)}}$, and $g^{(2)^{*}} E_{M}-E_{F_{1,2}}^{(2)}$ corresponds to $\mathbf{F}_{+}$under the isomorphism

$$
H^{0}\left(U, \Omega_{+}^{2}\left(\text { End } E_{M}\right)\right) \widetilde{\rightarrow} H^{1}\left(U^{\prime}, \mathcal{O}\left(\text { End } E_{F_{1,2}}\right) \otimes I_{1}^{2} / I_{1}^{3}\right) \text {. }
$$

The homomorphism

$$
\mathcal{O}\left(E_{M}\right)=g_{*} \mathcal{O}\left(E_{F_{1,2}^{(1,2}}\right) \rightarrow g_{*}^{1} \mathcal{O}\left(E_{F_{1,2}}\right) \otimes I_{1}^{2} / I_{1}^{3}=\Omega_{+}^{2}\left(E_{M}\right)
$$

is $-\mathbf{F}_{+}: \mathcal{O}\left(E_{M}\right) \rightarrow \Omega_{+}^{2}\left(E_{M}\right)$, and $\mathbf{F}_{-}$is (essentially) the curvature of $\tilde{\nabla}_{f^{(1)}}$.

The obstruction to extending $E_{A^{(n)}}$ now turns up as the obstruction

$$
\omega_{n}\left(\tilde{\nabla}_{f^{(n-1)}}\right) \in H^{1}\left(U_{1}^{\prime}, R_{f^{(n-1)}}^{2}\left(\text { End } E_{F_{1,2}}\right)\right)
$$

to extending $\tilde{\nabla}_{f^{(n-1)}}$ to a genuine relative connection on $E_{F_{1,2}^{(n)}}$. Indeed, given such an extension $\nabla_{f^{(n)}}$, it determines (by Proposition H(a)) an extension $\left(E_{F_{1,2}^{(n+1)}}, \tilde{\nabla}_{f^{(n)}}\right)$, and the pull-back of this object to $U^{\prime\{n+1\}}$ gives $\left(E_{F^{(n+1)}}, \tilde{\nabla}_{f^{(n)}}\right)$. By Proposition H(b), $\omega_{n}\left(\tilde{\nabla}_{f^{(n-1)}}\right)$ is the image of $\mathbf{F}\left(\nabla_{f^{(n-1)}}\right)$ under the connecting homomorphism from

$$
0 \rightarrow R_{f^{(n-1)}}^{2}\left(\text { End } E_{F_{1,2}}\right) \rightarrow \Omega_{M}^{2}\left(\text { End } E_{F_{1,2}^{(n-1)}}\right) \rightarrow \Omega_{f^{(n-1)}}^{2}\left(\text { End } E_{F_{1,2}^{(n-1)}}\right) \rightarrow 0 .
$$

For $n>2$, the sequence

$$
0 \rightarrow g_{*}^{1} R_{f^{(n-1)}}^{2} \rightarrow g_{*}^{1} \Omega_{M}^{2} \otimes I_{1}^{n-1} / I_{1}^{n} \rightarrow g_{*}^{1} \Omega_{M}^{3} \otimes I_{1}^{n-2} / I_{1}^{n-1}
$$

is exact, and the image of $\omega_{n}\left(\tilde{\nabla}_{f^{(n-1)}}\right)$ in $H^{1}\left(U^{\prime}, \Omega_{M}^{2}\left(\right.\right.$ End $\left.\left.E_{F_{1,2}}\right) \otimes I_{1}^{n-1} / I_{1}^{n}\right)$ coincides with that of $\mathbf{F}\left(\nabla_{f^{(n-1)}}\right)$ under the connecting homomorphism from

$$
0 \rightarrow \Omega_{M}^{2}\left(\text { End } E_{F_{1,2}}\right) \otimes I_{1}^{n-1} / I_{1}^{n} \rightarrow \Omega_{M}^{2}\left(\text { End } E_{F_{1,2}^{(n-1)}}\right) \rightarrow \Omega_{M}^{2}\left(\text { End } E_{F_{1,2}^{(n-2)}}\right) \rightarrow 0 .
$$

Since $\nabla_{f^{(n-1)}}$ is half-flat, $\Omega_{M}^{2}$ can be replaced by $\Omega_{-}^{2}$, so this image, in fact, lies in the kernel of

$$
H^{1}\left(U_{1}^{\prime}, \Omega_{-}^{2}\left(\text { End } E_{F_{1,2}}\right) \otimes I_{1}^{n-1} / I_{1}^{n-2}\right) \rightarrow H^{1}\left(U_{1}^{\prime}, \Omega_{M}^{3}\left(\text { End } E_{F_{1,2}}\right) \otimes I_{1}^{n-2} / I_{1}^{n-1}\right),
$$


which is isomorphic to

$$
H^{0}\left(U, \mathcal{O}\left(\odot^{n-1} S \otimes \odot^{n-1} S^{\prime}\right)\left(\text { End } E_{M}\right)[-1][-1]^{\prime}\right) .
$$

To identify the connecting map from (2.5.1), use the diagram

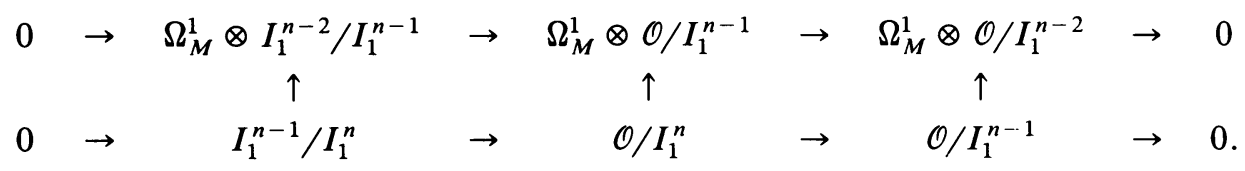

Tensoring through by $\mathcal{O}\left(E_{F_{1,2}^{(n-1)}}\right)$ and altering the vertical map to those induced by $\tilde{\nabla}_{f^{(n-1)}}$, it follows by induction that the map

$$
g_{*} \mathcal{O}\left(E_{F_{1,2}^{(n-2)}}\right) \rightarrow g_{*}^{1} \mathcal{O}\left(E_{F_{1,2}}\right) \otimes I_{1}^{n-1} / I_{1}^{n}
$$

can be viewed as

$$
\begin{aligned}
\mathcal{O}\left(E_{M}\right) & \supset g_{*} \mathcal{O}\left(E_{F_{1,2}^{(n-1)}}\right) \ni s \mapsto-\mathbf{F}_{+} \cdot \underbrace{\nabla \nabla \cdots \nabla}_{n-3} s \\
& \in \mathcal{O}\left(\odot^{n-1} S \otimes \odot^{n-3} S^{\prime}\right)\left(E_{M}\right)[-1]^{\prime} \subset \Omega_{+}^{2}\left(E_{M}\right) \otimes \odot^{n-3} \Omega_{M}^{1}
\end{aligned}
$$

(The symmetrized derivatives above are only defined on sections $s$ of $E_{M}$ with

$$
\mathbf{F}_{+} \cdot \underbrace{\nabla \nabla \cdots \nabla}_{n-4} s=0 \text {.) }
$$

Hence, by replacing $E_{F_{1,2}^{(n-1)}}$ by its endormorphism bundle, it follows that $\omega_{n}\left(\tilde{\nabla}_{f^{(n-1)}}\right)$ and, thus, $\omega_{n+1}\left(E_{A^{(n)}}\right)$ correspond to the section

$$
\begin{aligned}
\left(\nabla \nabla \cdots \nabla \mathbf{F}_{-}\right) \cdot F_{+}-\mathbf{F}_{+} & \cdot \nabla \nabla \cdots \nabla \mathbf{F}_{-} \\
& \in H^{0}\left(U, \mathcal{O}\left(\odot^{n-1} S \otimes \odot^{n-1} S^{\prime}\right)\left(\text { End } E_{M}\right)[-1][-1]^{\prime}\right) .
\end{aligned}
$$

If $E_{A}$ has rank $N$ and extends to $U^{\prime \prime}\left(N^{2}+2\right)$, then it extends to all orders. To prove this, observe that $g_{*} \mathcal{O}\left(E_{F_{1,2}^{(n+1)}}\right) \rightarrow g_{*} \mathcal{O}\left(E_{F_{1,2}^{(n)}}\right)$ is an isomorphism in a neighbourhood of a generic point of $U$ for $n>N$. For if it is not an isomorphism for $n=1,2, \ldots, N$, then $g_{*} \mathcal{O}\left(E_{F_{1,2}^{(n+1)}}\right)=0$ for all $n \geqslant N$ by virtue of the decreasing rank of these direct images (which are all free in a neighbourhood of a generic point). If $g_{*} \mathcal{O}\left(E_{F_{1,2}^{(n+1)}}\right) \rightarrow$ $g_{*} \mathcal{O}\left(E_{F_{1,2}^{(n)}}\right) \neq 0$ is an isomorphism for some $n$ between 1 and $N$, then $\nabla$ on $E_{M}$ restricts to a connection on $E_{M}^{\prime}:=g_{*} E_{F_{1,2}^{(n+1)}}$ via

$$
g_{*} \mathcal{O}\left(E_{F_{1,2}^{(n+1)}}\right) \rightarrow g_{*} \Omega_{M}^{1}\left(E_{F^{(n)} 1,2}\right) \simeq \Omega_{M}^{1}\left(E_{M}^{\prime}\right),
$$

and, moreover, the restricted connection is anti-self-dual. $E_{A}$ is now expressed as an extension $0 \rightarrow E_{A}^{\prime} \rightarrow E_{A} \rightarrow Q_{A} \rightarrow 0$, where $Q_{A}$ denotes the quotient. The homomorphism $E_{A}^{\prime} \rightarrow E_{A}$ extends to $U^{\prime \prime(n+1)}$, and, therefore, so does $Q_{A}$, thus giving the desired result by induction on $N$. Indeed, $g_{*} E_{F_{1,2}^{(m)}}=E_{M}^{\prime}$ for all $m$ sufficiently large. $\left(\right.$ For $N=1, g_{*} \mathcal{O}\left(E_{F_{1,2}^{(2)}}\right)=0$ or $\mathbf{F}_{+}=0$.)

If one now replaces $E_{A}$ by End $E_{A}$, it follows that

$$
g_{*} \Omega_{M}^{2}\left(\text { End } E_{F_{1,2}^{(n-1)}}\right) \rightarrow g_{*} \Omega_{M}^{2}\left(\text { End } E_{F_{1,2}^{(n-2)}}\right)
$$


is an isomorphism for $n>N^{2}+2$, but this estimate is improved to $n>N^{2}+1$ by factoring out the trivial line subbundle of the endormorphism bundle generated by the identity. The result now follows from (2.5.1) and the arguments leading to the identification of $\omega_{n+1}\left(E_{A^{(n)}}\right)$.

2.6. Examples and concluding remarks. Let $U \subset M$ be an open set whose intersection with every null line in $M$ is connected and simply connected. In order to construct examples of Yang-Mills bundles on $U$ with zero current, the first step, using the Witten-Isenberg-Yasskin-Green correspondence, is to construct $Q$-trivial bundles on $U^{\prime \prime} \subset A$. This in itself is not an easy problem, but it is by no means insurmountable.

The simplest case to deal with is that of rank 1 , where the condition of $Q$-triviality is equivalent to the vanishing of the first Chern class if $H^{2}(U, \mathbb{Z})=0$. Then if $L_{A}$ is a $Q$-trivial line bundle on $U^{\prime \prime}, L_{A}=\exp \phi_{A}$ for some $\phi_{A} \in H^{1}\left(U^{\prime \prime}, \mathcal{O}_{A}\right)$. If $U$ is, for example, Stein, the transform for cohomology gives

$$
H^{1}\left(U^{\prime \prime}, \mathcal{O}_{A}\right) \simeq \Gamma\left(U, \Omega_{M}^{1}\right) / d \Gamma\left(U, \mathcal{O}_{M}\right),
$$

and a 1-form $\phi_{M}$ corresponding to $\phi_{A}$ under this isomorphism is the same as a connection 1-form on $L_{M}$, the trivial line bundle on $U$. (More precisely, $\nabla$ on $L_{M}$ is given by $\nabla s=d s-\phi_{M} s$.) If $H^{2}(U, \mathbb{Z}) \neq 0$ or $U$ is not Stein, such $\phi_{M}$ may exist only locally, but whatever the case, the field is $-d \phi_{M}$ and the current is $-d * d \phi_{M}$. The class $\phi_{A}$ extends uniquely to $U^{\prime \prime(2)}$, and $\omega_{3}\left(\phi_{A^{(2)}}\right) \in H^{2}\left(U^{\prime \prime}, \mathcal{O}_{A}(-3,-3)\right)$ corresponds to $\frac{1}{12} d * d \phi_{M}$ under the transform for cohomology. Thus, if the current vanishes, $L_{A}=L_{+} \otimes L_{-}$for some (uniquely determined) self-dual and anti-self-dual line bundles $L_{ \pm}$.

The only $Q$-trivial line bundle on all of $A$ is trivial since $H^{1}\left(A, \mathcal{O}^{*}\right)=H^{2}(A, \mathbb{Z})=$ $\mathbb{Z} \oplus \mathbb{Z}$. In fact, it is not hard to show that the only $Q$-trivial bundles of arbitrary rank on all of $A$ are trivial.

For two $Q$-trivial bundles $E_{A}^{\prime}, E_{A}^{\prime \prime}$ on $U^{\prime \prime}$, an element

$$
\phi_{A} \in H^{1}\left(U^{\prime \prime}, \mathcal{O}\left(\operatorname{Hom}\left(E_{A}^{\prime}, E_{A}^{\prime \prime}\right)\right)\right)
$$

defines a third $Q$-trivial bundle $E_{A}$ as an extension $0 \rightarrow E_{A}^{\prime \prime} \rightarrow E_{A} \rightarrow E_{A}^{\prime} \rightarrow 0$. If $U$ is Stein, for example, the transform for cohomology gives

$$
\begin{aligned}
H^{1}\left(U^{\prime \prime}, \mathcal{O}(\operatorname{Hom}(\right. & \left.\left.\left.E_{A}^{\prime}, E_{A}^{\prime \prime}\right)\right)\right) \\
& \simeq \Gamma\left(U, \Omega_{M}^{1}\left(\operatorname{Hom}\left(E_{M}^{\prime}, E_{M}^{\prime \prime}\right)\right)\right) / \hat{\nabla} \Gamma\left(U, \mathcal{O}\left(\operatorname{Hom}\left(E_{M}^{\prime}, E_{M}^{\prime \prime}\right)\right)\right),
\end{aligned}
$$

where $\hat{\nabla}$ is the induced connection on $\operatorname{Hom}\left(E_{M}^{\prime}, E_{M}^{\prime \prime}\right)$. The local connection forms for $\nabla$ on $E_{M}$ are of the form

$$
\left(\begin{array}{cc}
A_{M}^{\prime \prime} & -\phi_{M} \\
0 & A_{M}^{\prime}
\end{array}\right),
$$

where $A_{M}^{\prime}$ and $A_{M}^{\prime \prime}$ are connection forms for $E_{M}^{\prime}$ and $E_{M}^{\prime \prime}$, respectively, and $\phi_{M} \in$ $\Gamma\left(U, \Omega_{M}^{1}\left(\operatorname{Hom}\left(E_{M}^{\prime}, E_{M}^{\prime \prime}\right)\right)\right)$ represents $\phi_{A}$ (a choice of $\phi_{M}$ corresponds to a choice of splitting $\left.E_{M} \simeq E_{M}^{\prime} \oplus E_{M}^{\prime \prime}\right)$. The field is

$$
\left(\begin{array}{cc}
\mathbf{F}^{\prime \prime} & -\hat{\nabla} \phi_{M} \\
0 & \mathbf{F}^{\prime}
\end{array}\right)
$$


and the current is

$$
\left(\begin{array}{cc}
\mathbf{J}^{\prime \prime} & -\left(\hat{\nabla} * \hat{\nabla} \phi-* \hat{\mathbf{F}} \wedge \phi_{M}\right) \\
0 & \mathbf{J}^{\prime}
\end{array}\right)
$$

The section $\hat{\nabla} * \hat{\nabla} \phi_{M}-* \hat{\mathbf{F}} \wedge \phi_{M}$ corresponds to

$$
12 \omega_{3}\left(\phi_{A^{(2)}}\right) \in H^{2}\left(U^{\prime \prime}, \mathcal{O}\left(\operatorname{Hom}\left(E_{A}^{\prime}, E_{A}^{\prime \prime}\right)\right)\right) \text {, }
$$

so $E_{A}$ extends to $U^{\prime \prime(3)}$ iff $E_{A}^{\prime}, E_{A}^{\prime \prime}$ and $\phi_{A}$ do (this can also be deduced directly from diagram-chasing on $U^{\prime \prime}$ ).

Nontrivial examples of such $\phi_{A}$ extending to $U^{\prime \prime(3)}$ are hard to find, even when $E_{A}^{\prime}$ and $E_{A}^{\prime \prime}$ are line bundles. There are, of course, many examples coming from the set of bundles on the product ( $:=$ subset of $P \times P^{*}$ corresponding to $U$ ) generated by the self-dual and anti-self-dual bundles and closed under the operations of tensor product and extensions. Indeed, it appears likely that every rank $N Q$-trivial bundle extending to $U^{\prime \prime}\left(N^{2}+2\right)$ belongs to this set. This is certainly the case for $N=1$ and 2 .

To give an example of a $Q$-trivial bundle extending to $U^{\prime \prime(3)}$, but not to all orders, the simplest case to try after line bundles is that of bundles of rank two. Here it is natural to consider extensions $0 \rightarrow L_{1} \rightarrow E_{A} \rightarrow L_{2} \rightarrow 0$, where $L_{1}$ and $L_{2}$ are line bundles. Since $E_{A}$ extends to $U^{\prime \prime(3)}$ only if det $E_{A}$ does, one can suppose that $L_{1}=L_{2}^{*}=L(p, q)$ for some line bundle $L$ with first Chern class zero. For simplicity, one can suppose that $L$ is in fact trivial. The condition of $Q$-triviality on $E_{A}$ requires that $p$ or $q$ be zero in order that $\left.E_{A}\right|_{Q_{x}}$ have zero second Chern class, so one is left with extensions of the form $0 \rightarrow \mathcal{O}(p, 0) \rightarrow \mathcal{O}\left(E_{A}\right) \rightarrow \mathcal{O}(-p, 0) \rightarrow 0$ (say). Extensions of this form correspond to elements of $H^{1}\left(U^{\prime \prime}, \mathcal{O}_{A}(2 p, 0)\right)$, and in order for $\left.E_{A}\right|_{Q}$ to be holomorphically trivial, it is necessary that $p$ be $\leqslant 0$.

For $p<-1$, every element of $H^{1}\left(U^{\prime \prime}, \mathcal{O}_{A}(2 p, 0)\right)$ extends (uniquely) to the product, thus giving a bundle with self-dual curvature. For $p=-1$, the transform for cohomology gives $H^{1}\left(U^{\prime \prime}, \mathcal{O}_{A}(-2,0)\right) \simeq H^{0}\left(U, \mathcal{O}_{M}[-1]^{\prime}\right)$; if $X_{A} \in H^{1}\left(U^{\prime \prime}, \mathcal{O}_{A}(-2,0)\right)$ and $X \in H^{0}\left(U, \mathcal{O}_{M}[-1]^{\prime}\right)$ is the corresponding section, then $\left.E_{A}\right|_{Q_{x}}$ is trivial iff $X(x) \neq 0$. Moreover, $\omega_{1}\left(X_{A}\right)$ corresponds to $\frac{1}{2} \square X$ under the isomorphism $H^{1}\left(U^{\prime \prime}, \mathcal{O}_{A}(-3,-1)\right) \simeq H^{0}\left(U, \mathcal{O}_{M}[-1][-2]^{\prime}\right)$ ( $\square$ is the conformally invariant wave operator). If $\omega_{1}\left(X_{A}\right)=0$, then $X_{A}$ extends to the product and the bundle $E_{A}$ is again self-dual. This leaves the case $\omega_{1}\left(X_{A}\right) \neq 0$, which turns out to be quite fruitful.

Suppose $X_{A} \in H^{1}\left(U^{\prime \prime}, \mathcal{O}_{A}(-2,0)\right)$ corresponds to $X \in H^{0}\left(U, \mathcal{O}_{M}[-1]^{\prime}\right)$ with $\square X \neq$ 0 . If $X_{A}^{\prime}$ is any other element of $H^{1}\left(U^{\prime \prime}, \mathcal{O}_{A}(-2,0)\right), E_{A} \simeq E_{A}^{\prime}$ iff $x=c X^{\prime}$ for some nonzero constant $c$. From the definition of $E_{A}, E_{M} \simeq S^{\prime *}$, and to give an explicit description of the field and current, it is most convenient to resort to spinor indices; an explanation of this notation can be found in [10].

In terms of the standard local trivializations of $S$ and $S^{\prime}$, the connection 1-forms are $\delta_{C^{\prime}}^{A^{\prime}} X^{-1} \nabla_{B^{\prime} C} X$, where $A^{\prime}, B^{\prime}$ are indices for End $S^{\prime *}$ and $C, C^{\prime}$ are indices for $\Omega_{M}^{1}=\mathcal{O}_{C C^{\prime}}$. The field is

$$
\left.\mathbf{F}_{-}=-\frac{1}{2} \boldsymbol{\delta}_{\left(C^{\prime \ell} D^{\prime}\right) B^{\prime}}^{A^{\prime}} X^{-1} \square X \text { and } \mathbf{F}_{+}=-X \nabla_{(C}^{A^{\prime}} \nabla D\right) B^{\prime} X^{-1},
$$

so the connection is self-dual iff $\square X=0$ (which has been ruled out) and anti-selfdual iff, locally, $X^{-1}=A+B_{a} x^{a}+C x_{a} x^{a}$ for some constants $A, B_{a}, C$ ( $a=$ 
$0,1,2,3)$. These anti-self-dual solutions are the (complex) instantons with topological charge (i.e., minus second Chern class) 1 . The current $\mathbf{J}$ is $-\delta_{\left(C^{\prime \ell} D^{\prime}\right) B^{\prime}}^{A^{\prime}} X^{2} \nabla_{D}^{C^{\prime}}\left(X^{-3} \square X\right)$, so the Yang-Mills equations $\nabla * \mathbf{F}=0$ are satisfied iff $X$ satisfies the so-called $\phi^{4}$-equation, $\square X=\lambda X^{3}$, where $\lambda \in \mathbb{C}$. This equation is familiar to physicists, and there are many known solutions; indeed, this construction has been known to physicists for some time as the $\phi^{4}$-Ansatz. (See Actor [1] for a more detailed description.) The commutator $\mathbf{F}_{-} \mathbf{F}_{+}-\mathbf{F}_{+} \mathbf{F}_{-}$corresponding to $\omega_{4}\left(E_{A^{(3)}}\right)$ vanishes iff the field is self-dual or anti-self-dual.

The connection is $\mathrm{SU}(2)$ on restriction to the real submanifold $U \cap S^{4}$ iff $\left.c X\right|_{S^{4} \cap U}$ is real for some constant $c \neq 0$. A large class of solutions of the $\phi^{4}$-equation has been found by twistor methods by using the embedding of $M$ in $\mathbb{P}\left(\wedge^{2} T\right)$ (Hughston and Hurd [17]). Specifically, let $X^{\mu}, \mu=0,1, \ldots, 5$, be homogeneous coordinates for $\mathbb{P}\left(\wedge^{2} T\right)$ such that $M$ is realized as the quadric $\rho_{\mu \nu} X^{\mu} X^{\nu}=0$, where $\rho_{\mu \nu}=\operatorname{diag}(-1,1,1,1,1,1)=\rho^{\mu \nu} ; S^{4} \hookrightarrow M$ is then $\left\{X^{\mu} \in M: X^{\mu}=\bar{X}^{\mu}\right\}$. If $R_{\mu \nu}$ is symmetric and satisfies $R_{\mu \nu} R_{\sigma \tau} \rho^{\nu \tau}=$ const $\rho_{\mu \sigma}$, the section $\left(R_{\mu \nu} X^{\mu} X^{\nu}\right)^{-1 / 2}$ of $\mathcal{O}(-1)$ is a solution of the $\phi^{4}$-equation on restriction to $M$. Of those solutions which are real on $S^{4}$, the singular sets are all of the form $S^{k} \hookrightarrow S^{4}$ for $k=-1,0,1,2,3$. The corresponding solutions of the Yang-Mills equations are strictly non-self-dual for $k \geqslant 0$, and the $k=-1$ (nonsingular) solutions are the anti-self-dual 1-instantons mentioned earlier. The $k=0$ solutions appear to be the so-called merons (Actor [1]). All $k \geqslant 0$ solutions have infinite action.

Although the ansatz has yet to produce any nonsingular strictly non-self-dual SU(2) solutions of the Yang-Mills equations on $S^{4}$, the above solutions of the $\phi^{4}$-equation do include nonsingular complex solutions on $S^{4}$, thus generating explicit $\operatorname{SL}(2, \mathbb{C})$ solutions of the Yang-Mills equations on $S^{4}$ with topological charge 1.

This paper concludes with a few brief remarks concerning certain current work which, to some extent, motivated the generality presented in Part I.

The generalization of the Ward correspondence to the non-self-dual case prompts one to ask if there is an analogous generalization of Penrose's "nonlinear graviton" construction [25], which locally produces all self-dual solutions of the Einstein vacuum equations. Le Brun [20] has pursued research in this direction and has succeeded in constructing the analogue of $A$ for a general complex spacetime with conformal metric; in [21] he carries this work further by constructing the analogue of $A^{(1)}$, and he has tentative candidates for the higher order neighbourhoods (private communications). In [8] Eastwood describes an approach to the constructions of " $A^{(n)}$ " for $n \geqslant 1$ which gives results similar to those of Le Brun-namely, the existence of an " $A^{(2)}$ " and the finding of (as yet unidentified) obstructions to the existence of a "third formal neighbourhood".

From the twistor point of view, one should hope that an analogue of $A^{(3)}$ can indeed be found in order that electromagnetic and other Yang-Mills fields on the spacetime can be described in terms of cohomology on the associated complex manifold, with curvature conditions on the spacetime corresponding to obstructions to constructing "formal neighbourhoods". There is much scope for further investigation. 


\section{REFERENCES}

1. A. Actor, Classical solutions of SU(2) Yang-Mills theories, Rev. Modern Phys. 51 (1979), 461-525.

2. M. F. Atiyah et al., Construction of instantons, Phys. Lett. A 65 (1978), 185-187.

3. R. Bott, Homogeneous vector bundles, Ann. of Math. (2) 56 (1957), 203-248.

4. J.-P. Bourguignon and H. B. Lawson, Jr., Stability and isolation phenomena for Yang-Mills fields, Comm. Math. Phys. 79 (1981), 189-230.

5. N. P. Buchdahl, On the relative de Rham sequence, Proc. Amer. Math. Soc. 87 (1983), 363-366.

6. M. G. Eastwood, Ambitwistors, Twistor Newsletter 9 (1979). ${ }^{1}$

7. __ Some remarks on non-abelian sheaf cohomology, Twistor Newsletter 12 (1981).

8. __ Formal thickenings of ambitwistors for curved space-time, Twistor Newsletter 17 (1984).

9. , The generalized Penrose-Ward transform, Math. Proc. Cambridge Philos. Soc. (to appear)

10. M. G. Eastwood, R. Penrose and R. O. Wells, Jr., Cohomology and massless fields, Comm. Math. Phys. 78 (1981), 305-351.

11. G. Fischer, Complex analytic geometry, Lecture Notes in Math., vol. 538, Springer, Berlin, 1976.

12. R. Godement, Topologie algébrique et théorie des faisceaux, Hermann, Paris, 1964.

13. H. Grauert and H. Kerner, Deformation von Singularitäten komplexen Räume, Math. Ann. 153 (1964), 236-260.

14. P. A. Griffiths, The extension problem in complex analysis. II, Amer. J. Math. 88 (1966), 266-446.

15. R. C. Gunning, Lectures on vector bundles over Riemann surfaces, Princeton Univ. Press, Princeton, N.J., 1967.

16. L. Hörmander, An introduction to complex analysis in several variables, North-Holland, Amsterdam and London, 1973.

17. L. P. Hughston and T. R. Hurd, Extensions of massless fields into $\mathbb{C P}^{5}$, Twistor Newsletter 12 (1981).

18. J. Isenberg and P. B. Yasskin, Twistor description of non-self-dual Yang-Mills fields, Complex Manifold Techniques in Theoretical Physics (D. E. Lerner and P. D. Sommers, eds.), Research Notes in Math., vol. 32, Pitman, San Francisco and London, 1979.

19. J. Isenberg, P. B. Yasskin and P. S. Green, Non-self-dual gauge fields, Phys. Lett. 878 (1978), 462-464.

20. C. Le Brun, Spaces of complex null geodesics in complex-Riemannian geometry, Trans. Amer. Math. Soc. 278 (1983), 209-231.

21. 6 (1982), 345-354.

22. Yu. I. Manin, Gauge fields and holomorphic geometry, J. Soviet Math. 21 (1983), 465-507.

23. Yu. I. Manin and G. M. Khenkin, Yang-Mills-Dirac equations as Cauchy-Riemann equations in twistor space, Soviet J. Nuclear Phys. 35 (6) (1982), 941-950.

24. R. Penrose, Solutions of the zero-rest-mass equations, J. Math. Phys. 10 (1969), 38-39.

25. __ Non-linear gravitons and curved twistor theory, Gen. Relativity Gravitation 7 (1976), 31-52.

26. R. Pool, Some applications of complex geometry to mathematical physics, $\mathrm{Ph}$. D. thesis, Rice Univ., 1981.

27. H. J. Reiffen, Das Lemma von Poincaré für holomorphe Differential-formen auf komplexen Räume, Math. Z. 101 (1967), 269-284.

28. R. S. Ward, On self-dual gauge fields, Phys. Lett. A 61 (1978), 289-295.

29. __ Ansätze for self-dual Yang-Mills fields, Comm. Math. Phys. 80 (1981), 563-574.

30. E. Witten, An interpretation of classical Yang-Mills theory, Phys. Lett. B 77 (1978), 394-398.

Department of Mathematics, Tulane University, New Orleans, Louisiana 70118

\footnotetext{
1“"Twistor Newsletter" is an informal series in preprint form distributed from the Mathematical Institute, Oxford.
} 